\title{
Gravity law in the Chinese highway freight transportation networks
}

\author{
Li Wang ${ }^{1}$, Jun-Chao Ma ${ }^{2,3}$, Zhi-Qiang Jiang ${ }^{1,3}$, Wanfeng Yan ${ }^{2,3}$ and Wei-Xing Zhou ${ }^{1,3,4^{*}}$ (D)
}

"Correspondence:
wxzhou@ecust.edu.cn
${ }^{1}$ School of Business, East China
University of Science and
Technology, Shanghai, China
${ }^{2}$ Zhicang Technologies, Beijing,
China
Full list of author information is
available at the end of the article

\begin{abstract}
The gravity law has been documented in many socioeconomic networks, which states that the flow between two nodes positively correlates with the strengths of the nodes and negatively correlates with the distance between the two nodes. However, such research on highway freight transportation networks (HFTNs) is rare. We construct the directed and undirected highway freight transportation networks between 338 Chinese cities using about 15.06 million truck transportation records in five months and test the traditional and modified gravity laws using GDP, population, and per capita GDP as the node strength. It is found that the gravity law holds over about two orders of magnitude for the whole sample, as well as the daily samples, except for the days around the Spring Festival during which the daily sample sizes are significantly small. Accordingly, the daily exponents of the gravity law are stable except during the Spring Festival period. The results also show that the gravity law has higher explanatory power for the undirected HFTNs than for the directed HFTNs. However, the traditional and modified gravity laws have comparable explanatory power.
\end{abstract}

Keywords: Gravity law; Highway freight transportation; Transportation network

\section{Main text}

Technological improvement and economic development promote the rapid growth of transportation systems globally and nationwide. In particular, over the past decades, the transportation systems in mainland China have gained remarkable achievements and the main transportation methods (aviation, railway, highway and shipping) form a rapidly expanding multiplex network. Since the Reform and Opening-up of China, it became a common sense for Chinese people that, "To be rich, build roads." The total length of highways was about 0.0808 million kilometers in early 1950 s and 0.890 million kilometers by the end of 1978. The construction of the first expressway started on 21 December 1984. The total highway length reached 4.85 million kilometers by the end of 2018 and the highway system contains national highways, provincial highways, county highways and countryside highways. The network includes 0.143 million kilometers expressways, making China the longest expressway network in the world.

The gravity law has been documented in many socioeconomic networks, in which locations (such as countries, regions and cities) are nodes and flows (such as products, mi-

(c) The Author(s) 2019. This article is licensed under a Creative Commons Attribution 4.0 International License, which permits use, sharing, adaptation, distribution and reproduction in any medium or format, as long as you give appropriate credit to the original author(s) and the source, provide a link to the Creative Commons licence, and indicate if changes were made. The images or other third party material in this article are included in the article's Creative Commons licence, unless indicated otherwise in a credit line to the material. If material is not included in the article's Creative Commons licence and your intended use is not permitted by statutory regulation or exceeds the permitted use, you will need to obtain permission directly from the copyright holder. To view a copy of this licence, visit http://creativecommons.org/licenses/by/4.0/. 
grants, and travelers) are edge weights. It states that the flow between two nodes positively correlates with the strengths of the nodes and negatively correlates with the distance between the two nodes. Inspired by Newton's law of gravity in physical sciences, gravity has been observed in human migration and mobility behavior [1-7], international trade flows [8-17], mobile phone communication flows [18-23], and so on. Some of these studies investigated the power-law decay of the flow with respect to the distance, while most others considered also node strengths.

As far as highway network is concerned, Jung, Wang and Stanley investigated the Korean highway network between the 30 largest cities [24]. They found that the traffic between two cities is positively proportional to the populations and negatively proportional to the distance, fitting exactly the traditional gravity law over two orders of magnitude. Kwon and Jung investigated the express bus flow in Korea consisting of 74 cities and 170 bus routes with 6692 operating buses per day and confirmed the presence of the gravity law [25]. Hong and Jung studied the urban bus networks of Korean cities and confirmed the presence of the gravity law [26].

In this work, we investigate a huge database about the freight highway transportation by trucks between 338 cities in mainland China. Although most studies dealt with undirected transportation networks [27, 28], we also consider directed transportation networks due to the availability of data. In our analysis, we consider the traditional gravity law, as well as the modified form of the gravity law in which the power-law exponents of the variables are not necessarily fixed.

\section{Data description}

The database we analyze was provided by a leading truck logistics company in China. The trucks transport freights between 338 cities in mainland China. The data covers the period from 1 January 2019 to 31 May 2019 and there are totally 15.06 million truck freight transportation records. Each record contains the origin and destination cities and the starting date of the transportation.

We construct the daily flow matrix $\mathbf{F}(t)=\left[F_{i j}(t)\right]_{338 \times 338}$ for each day $t$, where $F_{i j}(t)$ stands for the number of trucks with freights departing city $i$ for city $j$ on day $t$. Running trucks that do not load freights are not counted in. The flow matrix for the whole sample is thus

$$
\mathbf{F}=\sum_{t} \mathbf{F}(t)
$$

We note that $\mathbf{F}(t)$ and $\mathbf{F}$ are directed networks. When we consider the total truck flow $W_{i j}$, we obtain an undirected network:

$$
\mathbf{W}=\operatorname{triu}\left(\mathbf{F}+\mathbf{F}^{\mathbf{T}}\right),
$$

where triu denotes the upper triangle matrix operator and $\mathbf{T}$ is the transpose operator. By definition, we have

$$
W_{i i}=F_{i i}=0 .
$$

In other words, intracity transportation is not included in the data. 


\section{The traditional gravity model}

In the perspective of directed complex networks, the traditional gravity model states that the edge flow $F_{i j}$ between two nodes $i$ and $j$ takes the form of

$$
F_{i j}=C M_{i} M_{j} / d_{i j}^{2}
$$

for $i \neq j$, where $C$ is a constant, $d_{i j}$ stands for the distance between $i$ and $j$, and $M_{i}$ and $M_{j}$ stand respectively for the economic dimensions of the two nodes $i$ and $j$ that are being measured. For undirected transportation networks, we have

$$
W_{i j}=C M_{i} M_{j} / d_{i j}^{2}
$$

for $j>i$. In our analysis, we consider $M$ to be GDP $(G)$, population $(P)$ and per capita GDP $(G / P)$, respectively.

\subsection{GDP in the traditional gravity law}

\subsubsection{Whole transportation networks}

Figure 1 (a) shows the scatter plot of $F_{i j}$ with respect to $G_{i} G_{j} / d_{i j}^{2}$ for the directed network. When $M$ in Eq. (4) stands for GDP, the regression equation of the gravity law reads

$$
\lg F_{i j}=\alpha_{0}+\alpha_{1} \lg \left[G_{i} G_{j} / d_{i j}^{2}\right]+\epsilon,
$$

where $\epsilon$ is the error term. We obtain that $\alpha_{0}=1.383 \pm 0.004$ and $\alpha_{1}=0.612 \pm 0.004$, where the adjusted $R^{2}$ statistic is 0.476 , the $F$ statistic and $p$-value for the full model are respectively 85,246 and 0.000 , and an estimate of the error variance is 0.352 . We bin the data with respect to $F$ and illustrate the results in Fig. 1(b). We observe that there is a power-law dependence when $F$ is greater than about 100. A regression shows that $\alpha_{0}=1.489 \pm 0.025$ and $\alpha_{1}=0.965 \pm 0.017$ with their $p$-values being 0.0000 and 0.0000 . The adjusted $R^{2}$ statistic is 0.995 and the $F$ statistic and $p$-value for the full model are respectively 3296 and 0.0000 .

Figure 1 (c) shows the scatter plot of $W_{i j}$ with respect to $G_{i} G_{j} / d_{i j}^{2}$ for the undirected network. When $M$ in Eq. (5) stands for GDP, the regression equation of the gravity law reads

$$
\lg W_{i j}=\alpha_{0}+\alpha_{1} \lg \left[G_{i} G_{j} / d_{i j}^{2}\right]+\epsilon
$$

We obtain that $\alpha_{0}=1.705 \pm 0.005$ and $\alpha_{1}=0.662 \pm 0.005$, where the adjusted $R^{2}$ statistic is 0.566 , the $F$ statistic and $p$-value for the full model are respectively 66,530 and 0.000 , and an estimate of the error variance is 0.309 . We bin the data with respect to $W$ and illustrate the results in Fig. 1(d). We observe that there is a power-law dependence when $W$ is greater than about 200. A regression shows that $\alpha_{0}=1.917 \pm 0.022$ and $\alpha_{1}=0.828 \pm 0.013$ with the $p$-values being 0.0000 and 0.0000 . The adjusted $R^{2}$ statistic is 0.996 and the $F$ statistic and $p$-value for the full model are respectively 3912 and 0.0000 .

We find that, for the scatter plots, the adjusted $R^{2}$ statistic for the undirected HFTN (0.566) is greater than that for the directed HFTN (0.476). This result is visible in Fig. 1, which shows that the scatter plot is thinner for $W_{i j}$ when compared with the one for $F_{i j}$. 

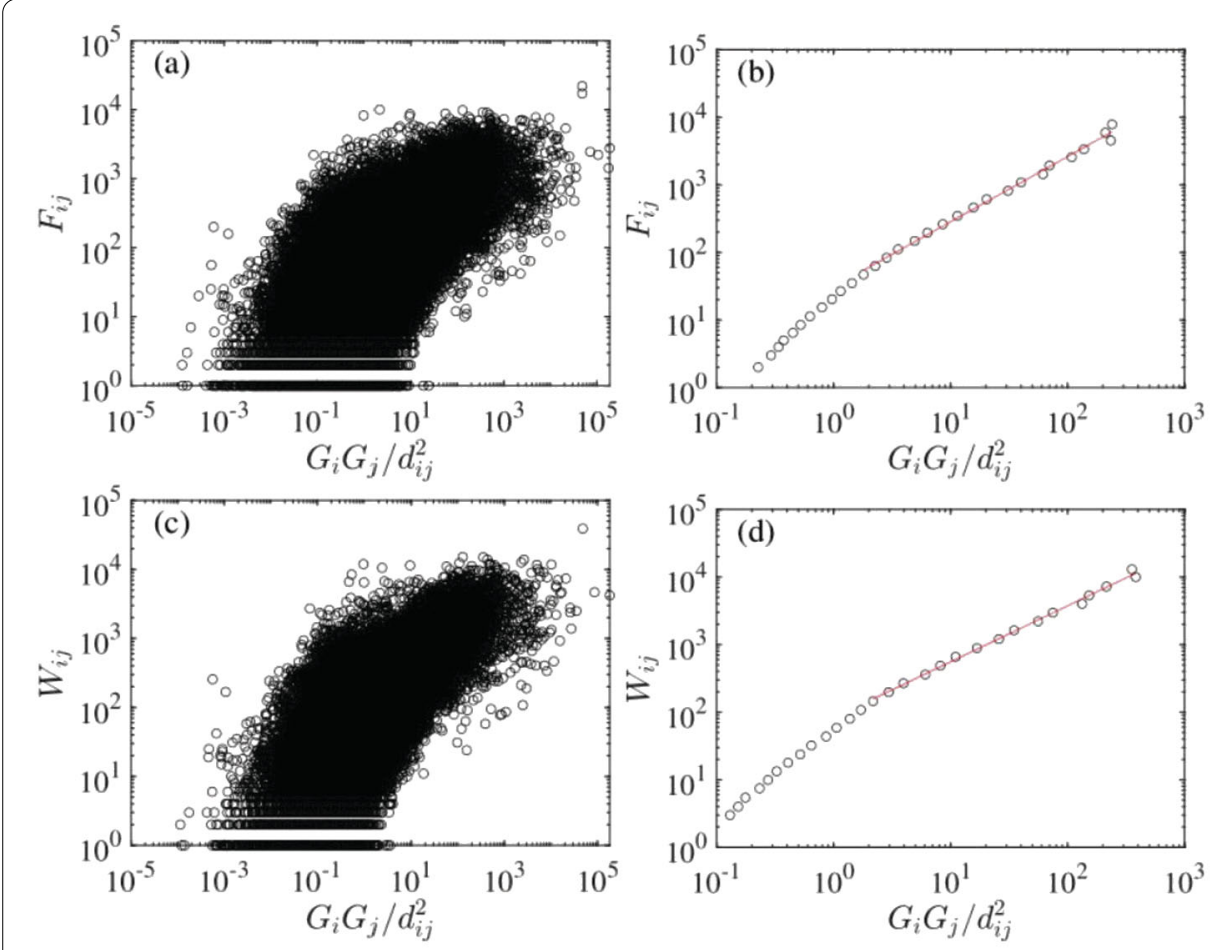

Figure 1 Testing the traditional gravity law with GDP for the directed and undirected freight highway transportation networks constructed over five months. (a) Scatter plot of $F_{i j}$ with respect to $G_{i} G_{j} / d_{i j}^{2}$ for the directed network. (b) Binning plot of $F_{i j}$ with respect to $G_{i} G_{j} / d_{i j}^{2}$ for the directed network. (c) Scatter plot of $W_{i j}$ with respect to $G_{i} G_{j} / d_{i j}^{2}$ for the undirected network. (d) Binning plot of $W_{i j}$ with respect to $G_{i} G_{j} / d_{i j}^{2}$ for the undirected network

\subsubsection{Daily transportation networks}

We now test the gravity law with daily directed and undirected freight highway transportation networks. We find that most of the daily networks exhibit the gravity law. As an example, the results for the directed network on 15 January 2019 are illustrated in Fig. 2(a). Regression of Eq. (6) for the scatter data points gives that $\alpha_{0}=0.196 \pm 0.006$ and $\alpha_{1}=0.194 \pm 0.005$, where the adjusted $R^{2}$ statistic is 0.191 , the $F$ statistic and $p$ value for the full model are respectively 6779 and 0.000 , and an estimate of the error variance is 0.129 . Regression for the binning data points shows that $\alpha_{0}=-0.257 \pm 0.130$ and $\alpha_{1}=0.824 \pm 0.080$, where the adjusted $R^{2}$ statistic is 0.947 , the $F$ statistic and $p$-value for the full model are respectively 449 and 0.000 , and an estimate of the error variance is 0.007 . The results for the undirected network on 15 January 2019 are illustrated in Fig. 2(b). Regression of Eq. (7) for the scatter data points gives that $\alpha_{0}=0.261 \pm 0.007$ and $\alpha_{1}=0.268 \pm 0.006$, where the adjusted $R^{2}$ statistic is 0.281 , the $F$ statistic and $p$ value for the full model are respectively 8246 and 0.000 , and an estimate of the error variance is 0.142 . Regression for the binning data points shows that $\alpha_{0}=-0.047 \pm 0.097$ and $\alpha_{1}=0.801 \pm 0.068$, where the adjusted $R^{2}$ statistic is 0.959 , the $F$ statistic and $p$-value for the full model are respectively 583 and 0.000 , and an estimate of the error variance is 0.006 .

However, we find that the daily networks around the Chinese New Year (5 February 2019) do not exhibit the gravity law. The transportation flow decreased significantly during 

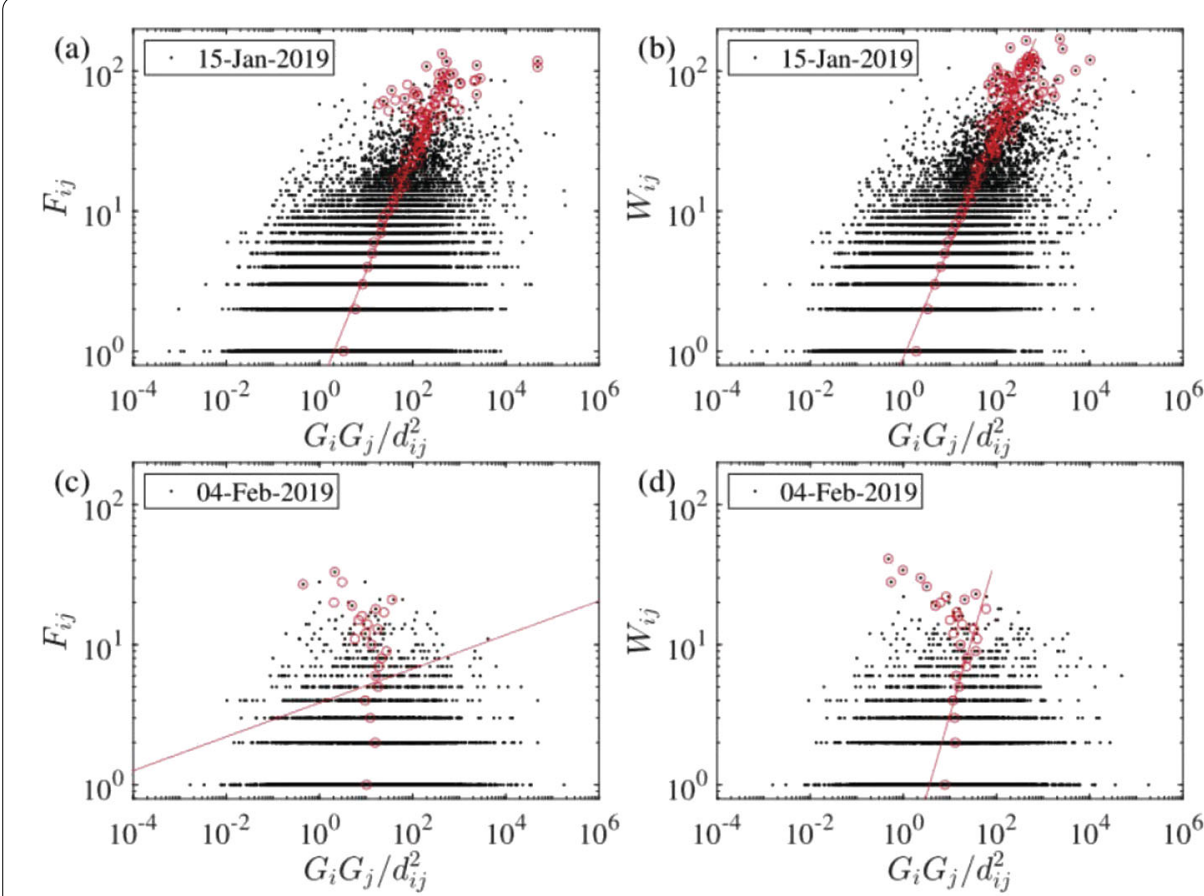

Figure 2 Testing the traditional gravity law with GDP for the daily directed and undirected freight highway transportation networks. (a) The directed network on 15 January 2019. (b) The directed network on 15 January 2019. (c) The directed network on 4 February 2019. (d) The undirected network on 4 February 2019

the Spring Festival because most of the truck drivers returned home to gather with their families and most companies were also closed. As an example, the results for the directed network on 4 February 2019 are illustrated in Fig. 2(c). Regression of Eq. (6) for the scatter data points gives that $\alpha_{0}=0.167 \pm 0.011$ and $\alpha_{1}=0.014 \pm 0.008$, where the adjusted $R^{2}$ statistic is 0.003 , the $F$ statistic and $p$-value for the full model are respectively 13 and 0.000 , and an estimate of the error variance is 0.076 . Regression for the binning data points shows that $\alpha_{0}=0.585 \pm 1.437$ and $\alpha_{1}=0.121 \pm 1.247$, where the adjusted $R^{2}$ statistic is 0.005 , the $F$ statistic and $p$-value for the full model are respectively 0.047 and 0.833 , and an estimate of the error variance is 0.118 . The results for the undirected network on 15 January 2019 are illustrated in Fig. 2(d). Regression of Eq. (7) for the scatter data points gives that $\alpha_{0}=$ $0.176 \pm 0.012$ and $\alpha_{1}=0.034 \pm 0.009$, where the adjusted $R^{2}$ statistic is 0.014 , the $F$ statistic and $p$-value for the full model are respectively 62 and 0.000 , and an estimate of the error variance is 0.085 . Regression for the binning data points shows that $\alpha_{0}=-0.642 \pm 0.934$ and $\alpha_{1}=1.142 \pm 0.740$, where the adjusted $R^{2}$ statistic is 0.485 , the $F$ statistic and $p$-value for the full model are respectively 11 and 0.006 , and an estimate of the error variance is 0.063. All the four adjusted $R^{2}$ statistics are close to zero, implying that the term $G_{i} G_{j} / d_{i j}^{2}$ does not have explanatory power for the transportation flow $F_{i j}$ or $W_{i j}$ and the gravity law is absent.

In Fig. 3, we present the evolution of the exponents $\alpha_{1}$ of daily directed and undirected freight highway transportation networks. In each case, the exponent fluctuates roughly around a constant. The cone peak or valley corresponds to the dates around the Spring Festival during which the gravity law does not hold. 

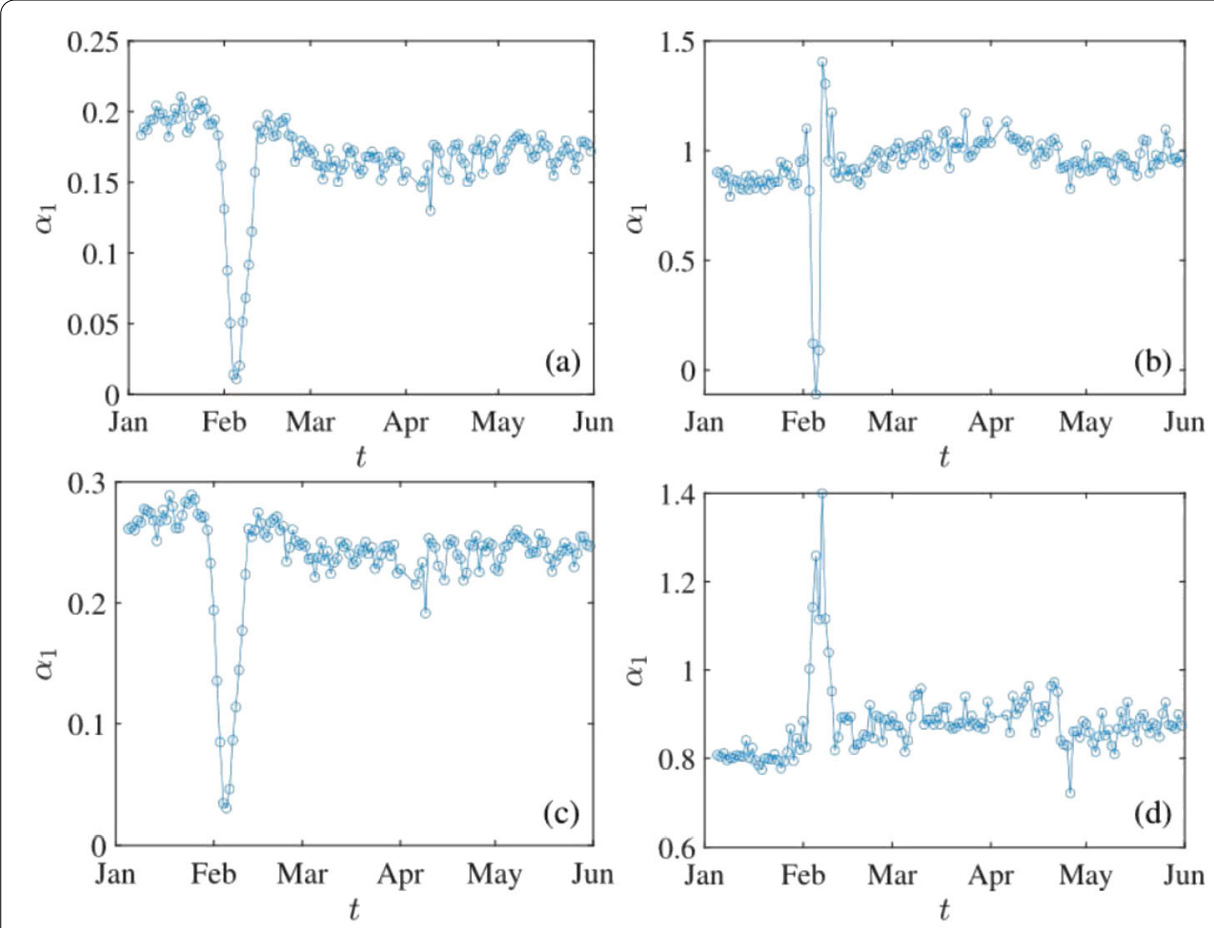

Figure 3 Evolution of the exponents $\alpha_{1}$ of daily directed and undirected freight highway transportation networks. (a) Scatter data of $F_{i j}$ with respect to $G_{i} G_{j} / d_{i j}^{2}$ for the directed network. (b) Binning data of $F_{i j}$ with respect to $G_{j} G_{j} / d_{i j}^{2}$ for the directed network. (c) Scatter data of $W_{i j}$ with respect to $G_{j} G_{j} / d_{i j}^{2}$ for the undirected network. (d) Binning data of $W_{i j}$ with respect to $G_{i} G_{j} / d_{i j}^{2}$ for the undirected network

\subsection{Population in the traditional gravity law}

\subsubsection{Whole transportation networks}

Figure 4(a) shows the scatter plot of $F_{i j}$ with respect to $P_{i} P_{j} / d_{i j}^{2}$ for the directed network. When $M$ in Eq. (4) stands for population, the regression equation of the gravity law reads

$$
\lg F_{i j}=\alpha_{0}+\alpha_{1} \lg \left[P_{i} P_{j} / d_{i j}^{2}\right]+\epsilon,
$$

where $\epsilon$ is the error term. We obtain that $\alpha_{0}=2.289 \pm 0.007$ and $\alpha_{1}=0.660 \pm 0.005$, where the adjusted $R^{2}$ statistic is 0.441 , the $F$ statistic and $p$-value for the full model are respectively 74,010 and 0.000 , and an estimate of the error variance is 0.376 . We bin the data with respect to $F$ and illustrate the results in Fig. 4(b). We observe that there is a power-law dependence when $F$ is greater than about 100. A regression shows that $\alpha_{0}=2.986 \pm 0.012$ and $\alpha_{1}=1.081 \pm 0.019$ with their $p$-values being 0.0000 and 0.0000 . The adjusted $R^{2}$ statistic is 0.995 and the $F$ statistic and $p$-value for the full model are respectively 3316 and 0.0000 .

Figure 4(c) shows the scatter plot of $W_{i j}$ with respect to $P_{i} P_{j} / d_{i j}^{2}$ for the undirected network. When $M$ in Eq. (5) stands for GDP, the regression equation of the gravity law reads

$$
\lg W_{i j}=\alpha_{0}+\alpha_{1} \lg \left[P_{i} P_{j} / d_{i j}^{2}\right]+\epsilon
$$

We obtain that $\alpha_{0}=2.683 \pm 0.009$ and $\alpha_{1}=0.717 \pm 0.006$, where the adjusted $R^{2}$ statistic is 0.525 , the $F$ statistic and $p$-value for the full model are respectively 56,394 and 0.000 , and an estimate of the error variance is 0.338 . We bin the data with respect to $W$ and illustrate 

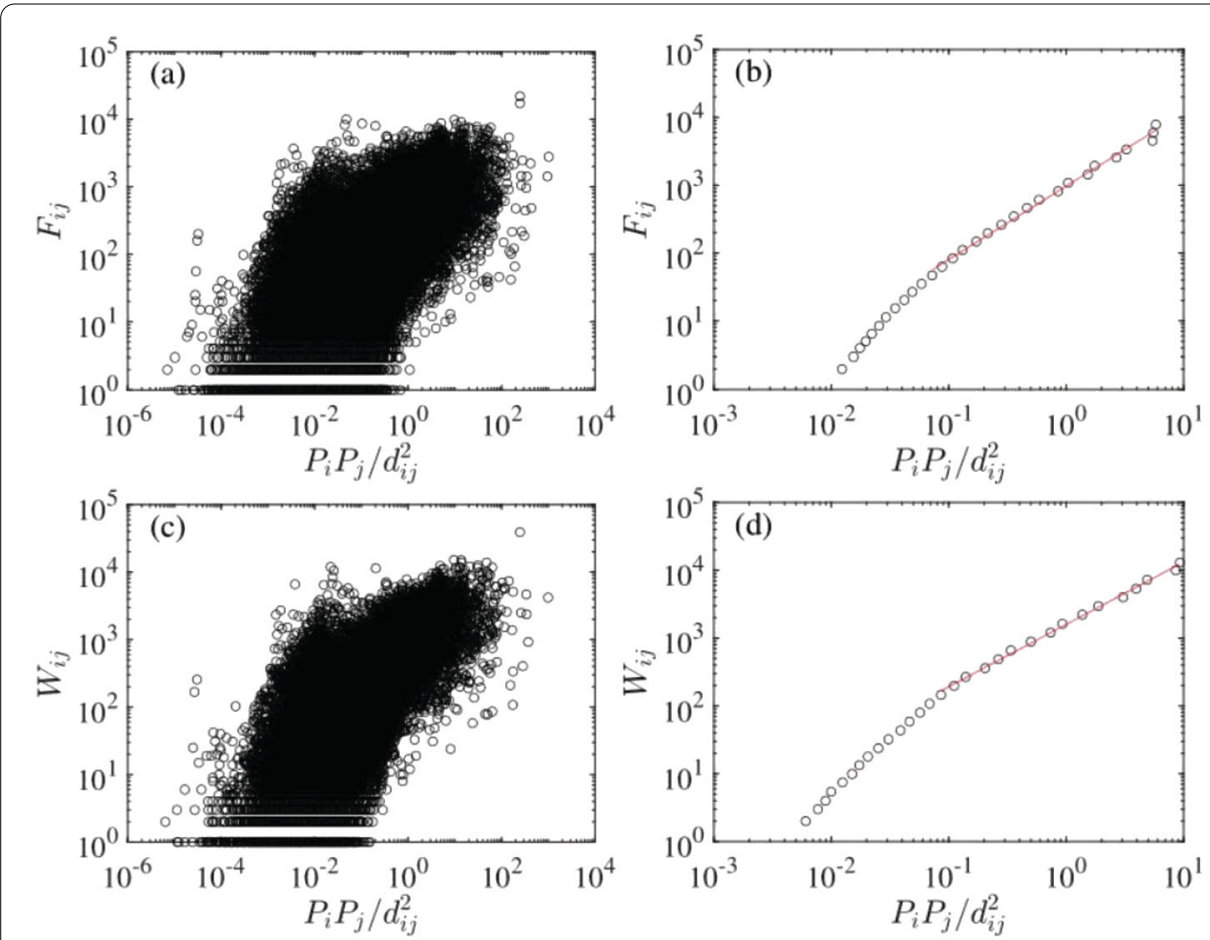

Figure 4 Testing the traditional gravity law with population for the directed and undirected freight highway transportation networks constructed over five months. (a) Scatter plot of $F_{i j}$ with respect to $P_{i} P_{j} / d_{i j}^{2}$ for the directed network. (b) Binning plot of $F_{i j}$ with respect to $P_{i} P_{j} / d_{i j}^{2}$ for the directed network. (c) Scatter plot of $W_{i j}$ with respect to $P_{i} P_{j} / d_{i j}^{2}$ for the undirected network. (d) Binning plot of $W_{i j}$ with respect to $P_{i} P_{j} / d_{i j}^{2}$ for the undirected network

the results in Fig. 4(d). We observe that there is a power-law dependence when $W$ is greater than about 200. A regression shows that $\alpha_{0}=3.202 \pm 0.009$ and $\alpha_{1}=0.919 \pm 0.013$ with the $p$-values being 0.0000 and 0.0000 . The adjusted $R^{2}$ statistic is 0.997 and the $F$ statistic and $p$-value for the full model are respectively 4647 and 0.0000 .

We find that, for the scatter plots, the adjusted $R^{2}$ statistic for the undirected HFTN $(0.525)$ is greater than that for the directed HFTN (0.441). This result is visible in Fig. 4, which shows that the scatter plot is thinner for $W_{i j}$ when compared with the one for $F_{i j}$.

\subsubsection{Daily transportation networks}

We now test the gravity law with daily directed and undirected freight highway transportation networks. We find that most of the daily networks exhibit the gravity law. As an example, the results for the directed network on 15 January 2019 are illustrated in Fig. 5(a). Regression of Eq. (8) for the scatter data points gives that $\alpha_{0}=0.491 \pm 0.005$ and $\alpha_{1}=0.206 \pm 0.005$, where the adjusted $R^{2}$ statistic is 0.176 , the $F$ statistic and $p$ value for the full model are respectively 6128 and 0.000 , and an estimate of the error variance is 0.131 . Regression for the binning data points shows that $\alpha_{0}=1.043 \pm 0.040$ and $\alpha_{1}=0.939 \pm 0.109$, where the adjusted $R^{2}$ statistic is 0.927 , the $F$ statistic and $p$-value for the full model are respectively 317 and 0.000, and an estimate of the error variance is 0.010 . The results for the undirected network on 15 January 2019 are illustrated in Fig. 5(b). Regression of Eq. (9) for the scatter data points gives that $\alpha_{0}=0.663 \pm 0.007$ and $\alpha_{1}=0.283 \pm 0.007$, where the adjusted $R^{2}$ statistic is 0.256 , the $F$ statistic and $p$ - 

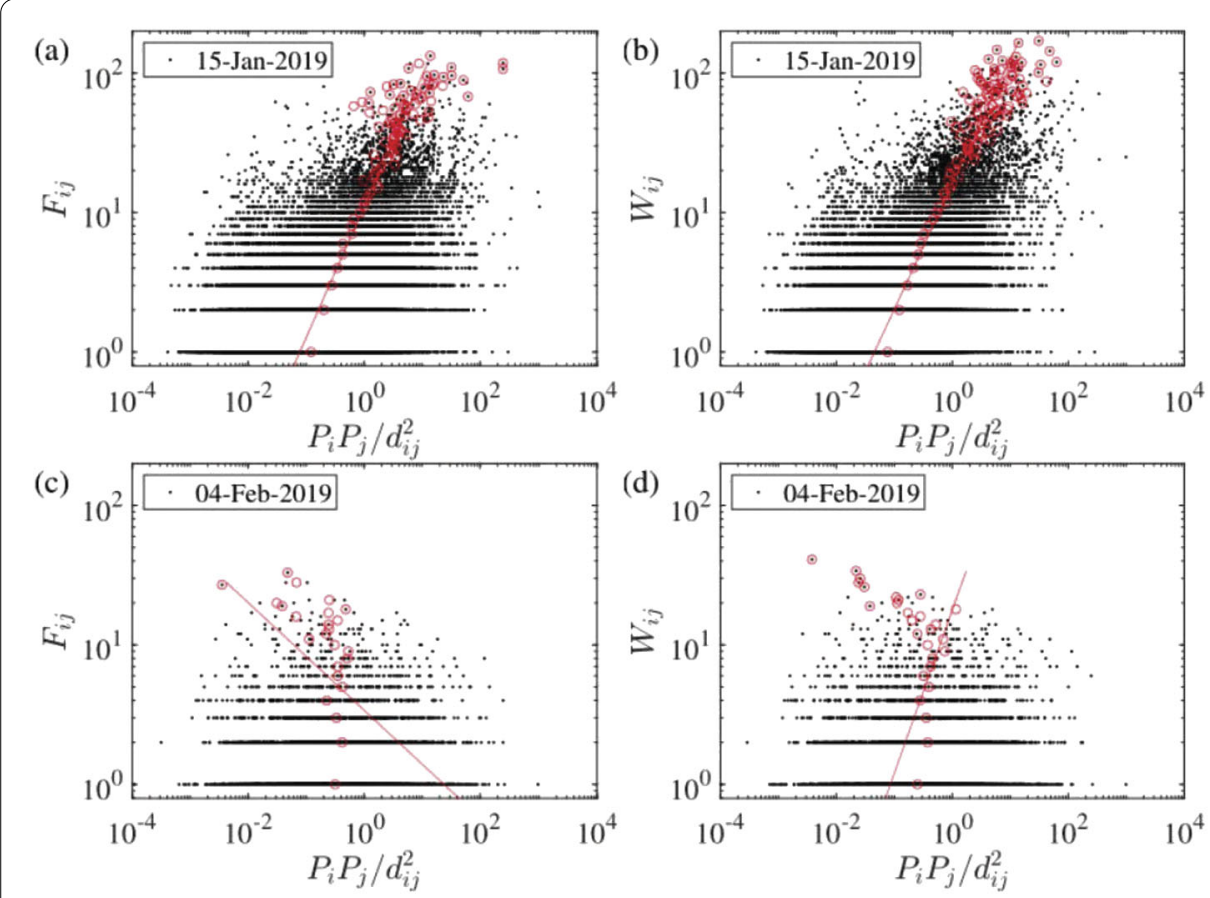

Figure 5 Testing the traditional gravity law with population for the daily directed and undirected freight highway transportation networks. (a) The directed network on 15 January 2019. (b) The directed network on 15 January 2019. (c) The directed network on 4 February 2019. (d) The undirected network on 4 February 2019

value for the full model are respectively 7253 and 0.000 , and an estimate of the error variance is 0.147 . Regression for the binning data points shows that $\alpha_{0}=1.203 \pm 0.038$ and $\alpha_{1}=0.896 \pm 0.087$, where the adjusted $R^{2}$ statistic is 0.947 , the $F$ statistic and $p$-value for the full model are respectively 448 and 0.000 , and an estimate of the error variance is 0.007 .

However, we find that the daily networks around the Chinese New Year (5 February 2019) do not exhibit the gravity law. The transportation flow decreased significantly during the Spring Festival because most of the truck drivers returned home to gather with their families and most companies were also closed. As an example, the results for the directed network on 4 February 2019 are illustrated in Fig. 5(c). Regression of Eq. (8) for the scatter data points gives that $\alpha_{0}=0.182 \pm 0.009$ and $\alpha_{1}=0.001 \pm 0.008$, where the adjusted $R^{2}$ statistic is 0.000 , the $F$ statistic and $p$-value for the full model are respectively 0.072 and 0.789 , and an estimate of the error variance is 0.076 . Regression for the binning data points shows that $\alpha_{0}=0.531 \pm 0.645$ and $\alpha_{1}=-0.386 \pm 1.222$, where the adjusted $R^{2}$ statistic is 0.047 , the $F$ statistic and $p$-value for the full model are respectively 0.496 and 0.497 , and an estimate of the error variance is 0.113 . The results for the undirected network on 15 January 2019 are illustrated in Fig. 5(d). Regression of Eq. (9) for the scatter data points gives that $\alpha_{0}=0.222 \pm 0.010$ and $\alpha_{1}=0.022 \pm 0.010$, where the adjusted $R^{2}$ statistic is 0.005 , the $F$ statistic and $p$-value for the full model are respectively 20.5 and 0.000 , and an estimate of the error variance is 0.086 . Regression for the binning data points shows that $\alpha_{0}=1.251 \pm 0.540$ and $\alpha_{1}=1.171 \pm 1.271$, where the adjusted $R^{2}$ statistic is 0.251 , the $F$ statistic and $p$-value for the full model are respectively 4.03 and 0.068 , and an estimate of the error variance is 0.092 . All the four adjusted $R^{2}$ statistics are close to zero, implying 

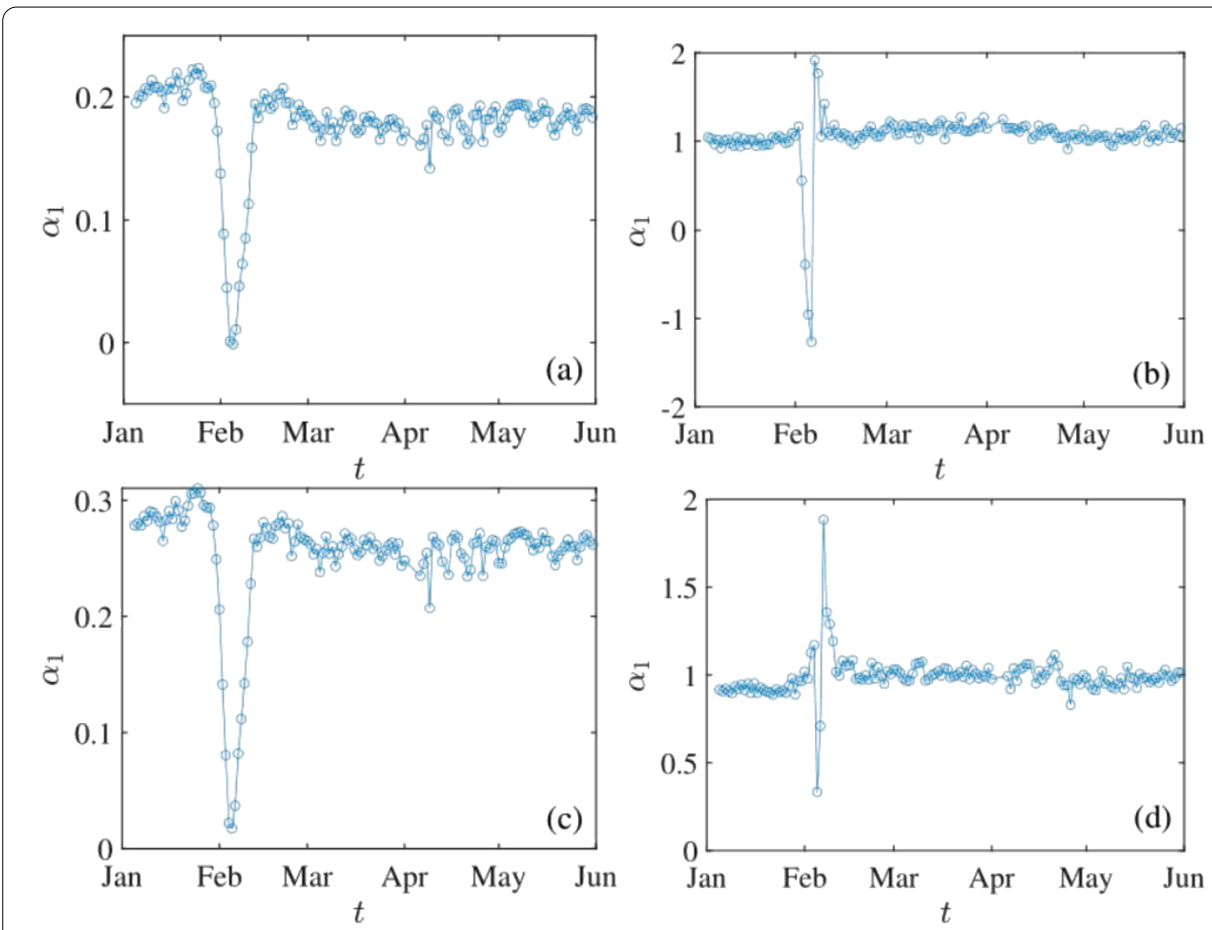

Figure 6 Evolution of the exponents $\alpha_{1}$ of daily directed and undirected freight highway transportation networks. (a) Scatter data of $F_{i j}$ with respect to $P_{i} P_{j} / d_{i j}^{2}$ for the directed network. (b) Binning data of $F_{i j}$ with respect to $P_{i} P_{j} / d_{i j}^{2}$ for the directed network. (c) Scatter data of $W_{i j}$ with respect to $P_{i} P_{j} / d_{i j}^{2}$ for the undirected network. (d) Binning data of $W_{i j}$ with respect to $P_{i} P_{j} / d_{i j}^{2}$ for the undirected network

that the term $P_{i} P_{j} / d_{i j}^{2}$ does not have explanatory power for the transportation flow $F_{i j}$ or $W_{i j}$ and the gravity law is absent.

In Fig. 6, we present the evolution of the exponents $\alpha_{1}$ of daily directed and undirected freight highway transportation networks. In each case, the exponent fluctuates roughly around a constant. The cone peak or valley corresponds to the dates around the Spring Festival during which the gravity law does not hold.

\subsection{Per capita GDP in the traditional gravity law}

\subsubsection{Whole transportation networks}

Figure 7(a) shows the scatter plot of $F_{i j}$ with respect to $\left(G_{i} / P_{i}\right)\left(G_{j} / P_{j}\right) / d_{i j}^{2}$ for the directed network. When $M$ in Eq. (4) stands for per capita GDP, the regression equation of the gravity law reads

$$
\lg F_{i j}=\alpha_{0}+\alpha_{1} \lg \left[\left(G_{i} / P_{i}\right)\left(G_{j} / P_{j}\right) / d_{i j}^{2}\right]+\epsilon,
$$

where $\epsilon$ is the error term. We obtain that $\alpha_{0}=4.772 \pm 0.030$ and $\alpha_{1}=0.672 \pm 0.006$, where the adjusted $R^{2}$ statistic is 0.328 , the $F$ statistic and $p$-value for the full model are respectively 45,778 and 0.000 , and an estimate of the error variance is 0.452 . We bin the data with respect to $F$ and illustrate the results in Fig. 7(b). We observe that there is a power-law dependence when $F$ is greater than about 100. A regression shows that $\alpha_{0}=8.961 \pm 0.145$ and $\alpha_{1}=1.496 \pm 0.035$ with their $p$-values being 0.0000 and 0.0000 . The adjusted $R^{2}$ statistic is 0.990 and the $F$ statistic and $p$-value for the full model are respectively 1830 and 0.0000 . 

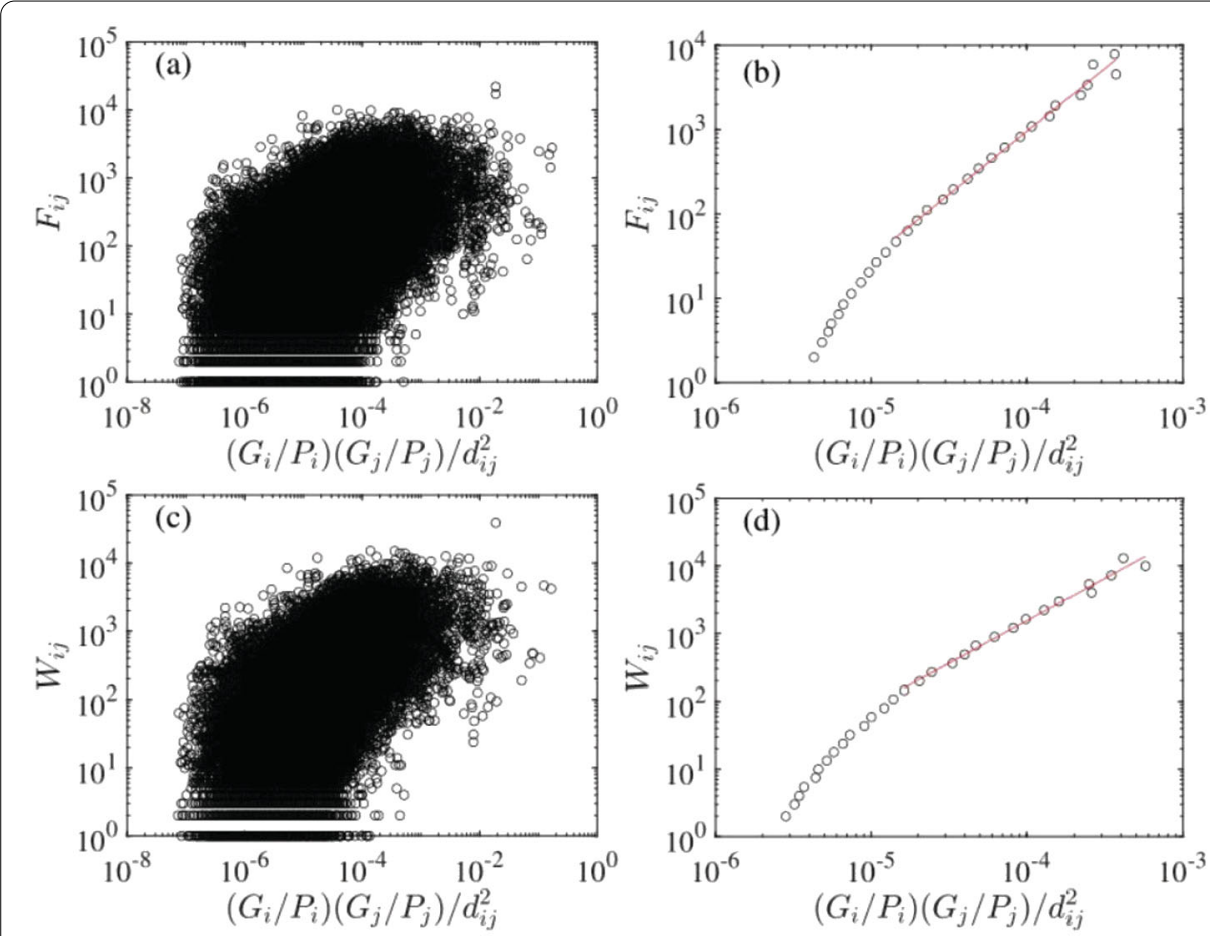

Figure 7 Testing the traditional gravity law with per capita GDP for the directed and undirected freight highway transportation networks constructed over five months. (a) Scatter plot of $F_{i j}$ with respect to $\left(G_{j} / P_{i}\right)\left(G_{j} / P_{j}\right) / d_{i j}^{2}$ for the directed network. (b) Binning plot of $F_{i j}$ with respect to $\left(G_{j} / P_{j}\right)\left(G_{j} / P_{j}\right) / d_{i j}^{2}$ for the directed network. (c) Scatter plot of $W_{i j}$ with respect to $\left(G_{i} / P_{i}\right)\left(G_{j} / P_{j}\right) / d_{i j}^{2}$ for the undirected network. (d) Binning plot of $W_{i j}$ with respect to $\left(G_{i} / P_{i}\right)\left(G_{j} / P_{j}\right) / d_{i j}^{2}$ for the undirected network

Figure 7 (c) shows the scatter plot of $W_{i j}$ with respect to $\left(G_{i} / P_{i}\right)\left(G_{j} / P_{j}\right) / d_{i j}^{2}$ for the undirected network. When $M$ in Eq. (5) stands for per capita GDP, the regression equation of the gravity law reads

$$
\lg W_{i j}=\alpha_{0}+\alpha_{1} \lg \left[\left(G_{i} / P_{i}\right)\left(G_{j} / P_{j}\right) / d_{i j}^{2}\right]+\epsilon .
$$

We obtain that $\alpha_{0}=5.416 \pm 0.040$ and $\alpha_{1}=0.740 \pm 0.008$, where the adjusted $R^{2}$ statistic is 0.387 , the $F$ statistic and $p$-value for the full model are respectively 32,211 and 0.000 , and an estimate of the error variance is 0.437 . We bin the data with respect to $W$ and illustrate the results in Fig. 7(d). We observe that there is a power-law dependence when $W$ is greater than about 200. A regression shows that $\alpha_{0}=8.215 \pm 0.143$ and $\alpha_{1}=1.257 \pm 0.035$ with the $p$-values being 0.0000 and 0.0000 . The adjusted $R^{2}$ statistic is 0.988 and the $F$ statistic and $p$-value for the full model are respectively 1266 and 0.0000 .

We find that, for the scatter plots, the adjusted $R^{2}$ statistic for the undirected HFTN (0.387) is greater than that for the directed HFTN (0.328). This result is visible in Fig. 7, which shows that the scatter plot is thinner for $W_{i j}$ when compared with the one for $F_{i j}$.

\subsubsection{Daily transportation networks}

We now test the gravity law with daily directed and undirected freight highway transportation networks. We find that most of the daily networks exhibit the gravity law. As an example, the results for the directed network on 15 January 2019 are illustrated in 

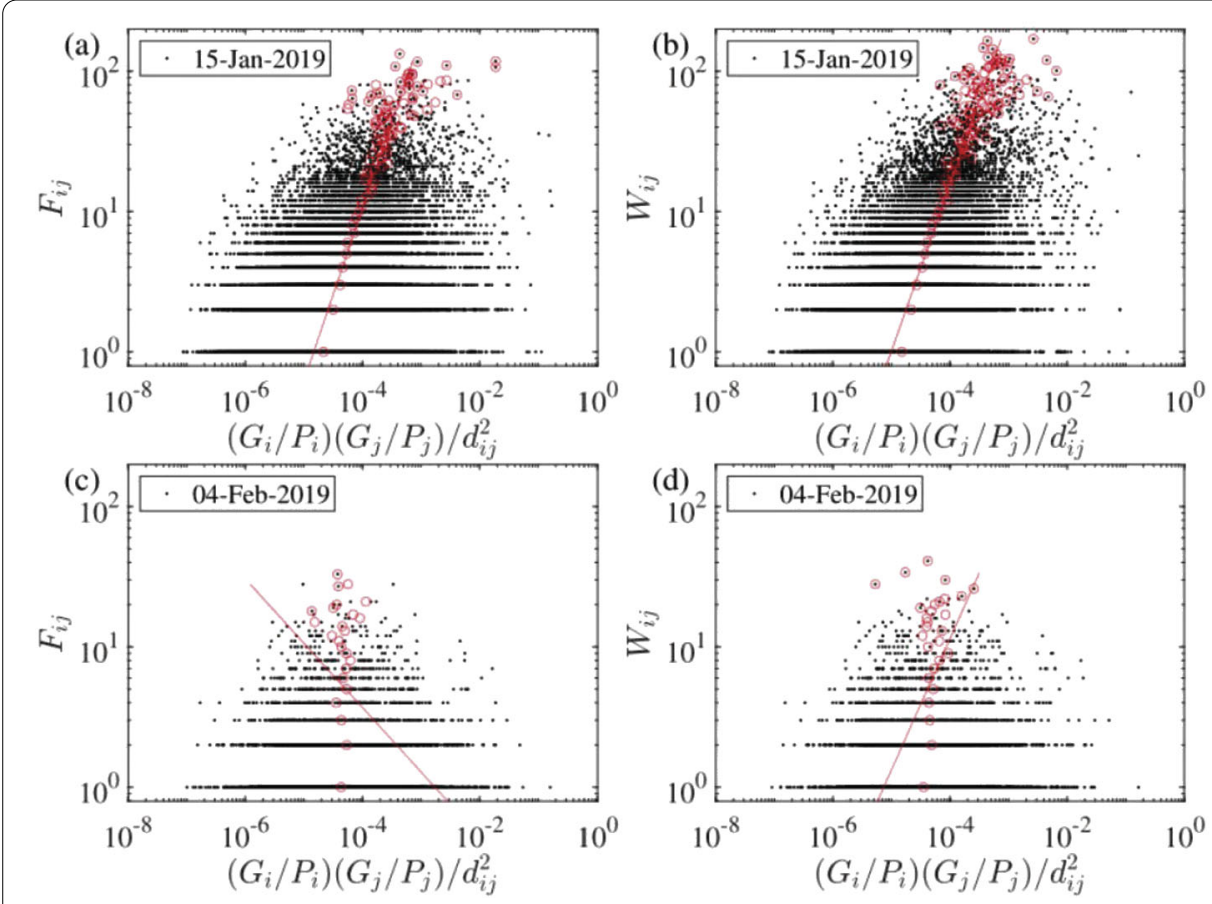

Figure 8 Testing the traditional gravity law with per capita GDP for the daily directed and undirected freight highway transportation networks. (a) The directed network on 15 January 2019. (b) The directed network on 15 January 2019. (c) The directed network on 4 February 2019. (d) The undirected network on 4 February 2019

Fig. 8(a). Regression of Eq. (10) for the scatter data points gives that $\alpha_{0}=1.185 \pm 0.027$ and $\alpha_{1}=0.185 \pm 0.006$, where the adjusted $R^{2}$ statistic is 0.117 , the $F$ statistic and $p$ value for the full model are respectively 3788 and 0.000 , and an estimate of the error variance is 0.141 . Regression for the binning data points shows that $\alpha_{0}=5.933 \pm 0.624$ and $\alpha_{1}=1.227 \pm 0.156$, where the adjusted $R^{2}$ statistic is 0.913 , the $F$ statistic and $p$-value for the full model are respectively 262 and 0.000 , and an estimate of the error variance is 0.012 . The results for the undirected network on 15 January 2019 are illustrated in Fig. 8(b). Regression of Eq. (11) for the scatter data points gives that $\alpha_{0}=1.623 \pm 0.035$ and $\alpha_{1}=0.258 \pm 0.008$, where the adjusted $R^{2}$ statistic is 0.175 , the $F$ statistic and $p$ value for the full model are respectively 4465 and 0.000 , and an estimate of the error variance is 0.163 . Regression for the binning data points shows that $\alpha_{0}=5.947 \pm 0.611$ and $\alpha_{1}=1.188 \pm 0.148$, where the adjusted $R^{2}$ statistic is 0.917 , the $F$ statistic and $p$-value for the full model are respectively 275 and 0.000 , and an estimate of the error variance is 0.012 .

However, we find that the daily networks around the Chinese New Year (5 February 2019) do not exhibit the gravity law. The transportation flow decreased significantly during the Spring Festival because most of the truck drivers returned home to gather with their families and most companies were also closed. As an example, the results for the directed network on 4 February 2019 are illustrated in Fig. 8(c). Regression of Eq. (10) for the scatter data points gives that $\alpha_{0}=0.206 \pm 0.042$ and $\alpha_{1}=0.005 \pm 0.009$, where the adjusted $R^{2}$ statistic is 0.000 , the $F$ statistic and $p$-value for the full model are respectively 1.300 and 0.254 , and an estimate of the error variance is 0.076 . Regression for the binning data points shows that $\alpha_{0}=-1.266 \pm 11.353$ and $\alpha_{1}=-0.458 \pm 2.616$, where the adjusted $R^{2}$ statistic 

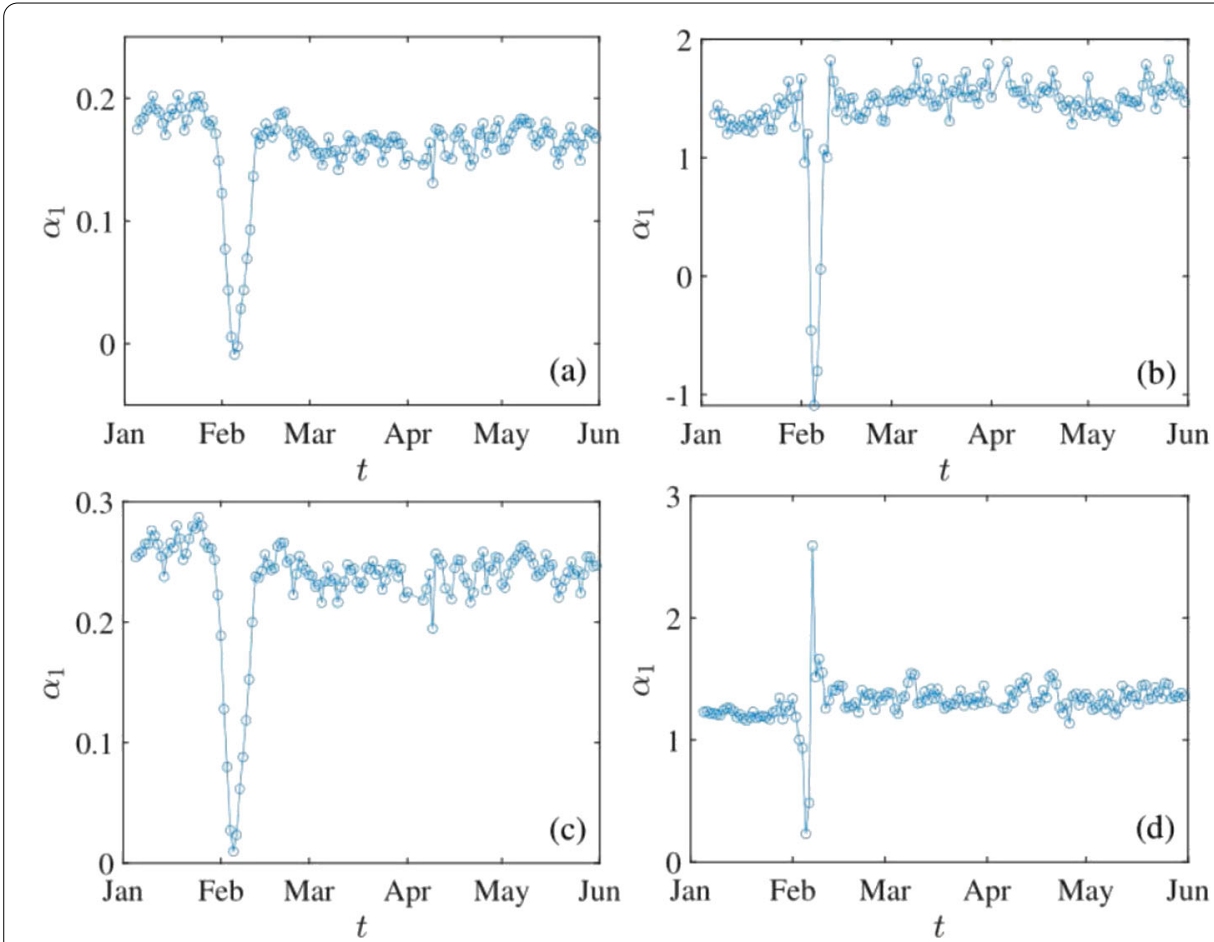

Figure 9 Evolution of the exponents $\alpha_{1}$ of daily directed and undirected freight highway transportation networks. (a) Scatter data of $F_{i j}$ with respect to $\left(G_{j} / P_{i}\right)\left(G_{j} / P_{j}\right) / d_{i j}^{2}$ for the directed network. (b) Binning data of $F_{i j}$ with respect to $\left(G_{j} / P_{i}\right)\left(G_{j} / P_{j}\right) / d_{i j}^{2}$ for the directed network. (c) Scatter data of $W_{i j}$ with respect to $\left(G_{i} / P_{i}\right)\left(G_{j} / P_{j}\right) / d_{i j}^{2}$ for the undirected network. (d) Binning data of $W_{i j}$ with respect to $\left(G_{i} / P_{i}\right)\left(G_{j} / P_{j}\right) / d_{i j}^{2}$ for the undirected network

is 0.015 , the $F$ statistic and $p$-value for the full model are respectively 0.153 and 0.704 , and an estimate of the error variance is 0.117 . The results for the undirected network on 15 January 2019 are illustrated in Fig. 8(d). Regression of Eq. (11) for the scatter data points gives that $\alpha_{0}=0.330 \pm 0.048$ and $\alpha_{1}=0.027 \pm 0.011$, where the adjusted $R^{2}$ statistic is 0.006 , the $F$ statistic and $p$-value for the full model are respectively 25.2 and 0.000 , and an estimate of the error variance is 0.086 . Regression for the binning data points shows that $\alpha_{0}=4.802 \pm 6.791$ and $\alpha_{1}=0.936 \pm 1.580$, where the adjusted $R^{2}$ statistic is 0.122 , the $F$ statistic and $p$-value for the full model are respectively 1.67 and 0.221 , and an estimate of the error variance is 0.108 . All the four adjusted $R^{2}$ statistics are close to zero, implying that the term $\left(G_{i} / P_{i}\right)\left(G_{j} / P_{j}\right) / d_{i j}^{2}$ does not have explanatory power for the transportation flow $F_{i j}$ or $W_{i j}$ and the gravity law is absent.

In Fig. 9, we present the evolution of the exponents $\alpha_{1}$ of daily directed and undirected freight highway transportation networks. In each case, the exponent fluctuates roughly around a constant. The cone peak or valley corresponds to the dates around the Spring Festival during which the gravity law does not hold.

\subsection{Relationship between exponents}

\subsubsection{GDP versus population}

It is well known that there is an anomalous power-law scaling between the GDP and population at the city level for many countries [29]:

$$
G_{i} \sim P_{i}^{\kappa}
$$


Figure 10 Power-law scaling between the GDP and population for the 338 Chinese cities in 2017

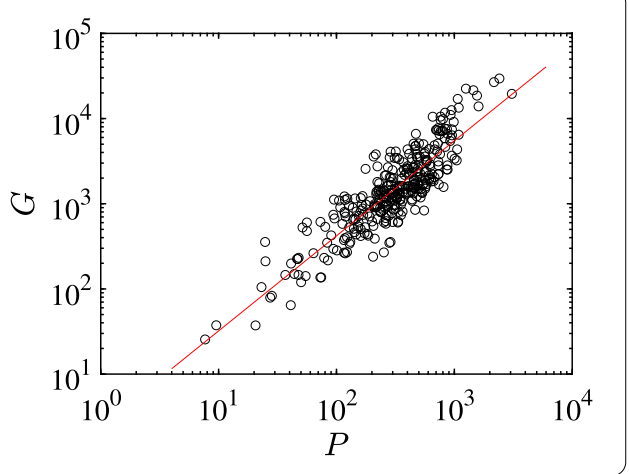

where the scaling exponent $\kappa$ fluctuates for different countries. Particularly, empirical evidence shows that $\kappa=1.15 \pm 0.08$ with $R^{2}=0.96$ for 295 Chinese cities in 2002 [29].

In Fig. 10, we show the GDP and population for the 338 Chinese cities in 2017 on log-log scales. It shows that there is a power-law scaling between $G$ and $P$. A linear regression of the following equation

$$
\lg G=\kappa_{0}+\kappa \lg P
$$

gives that $\kappa_{0}=0.391 \pm 0.083$ and $\kappa=1.116 \pm 0.033$, where the corresponding $p$-values are 0.0000 and 0.0000 , the adjusted $R^{2}$ statistic is 0.771 , the $F$ statistic and $p$-value for the full model are respectively 1132 and 0.0000 .

\subsubsection{Exponents of GDP and population}

From Eq. (6), we have $F_{i j} \sim\left(G_{i} G_{J}\right)_{G}^{\alpha}$. From Eq. (8), we have $F_{i j} \sim\left(P_{i} P_{j}\right)_{P}^{\alpha}$. From Eq. (7), we have $W_{i j} \sim\left(G_{i} G_{J}\right)_{G}^{\alpha}$. From Eq. (9), we have $W_{i j} \sim\left(P_{i} P_{j}\right)_{P}^{\alpha}$. Combining Eq. (12) and these two gravity laws for GDP and population, it is easy to obtain that

$$
\alpha_{G} \kappa=\alpha_{P}
$$

where $\alpha_{G}$ is $\alpha_{1}$ in the gravity model for GDP and $\alpha_{P}$ is $\alpha_{1}$ in the gravity model for population.

We test the relationship (14) with the exponents of the daily transportation networks. The data for the dates during 1 February 2019 to 9 February 2019 are excluded because their corresponding transportation networks do not exhibit the gravity law. In Fig. 11(a), the exponents $\alpha_{G}$ are those in Fig. 3(a) for the directed networks and Fig. 3(c) for the undirected networks, while the exponents $\alpha_{P}$ are those in Fig. 6(a) for the directed networks and Fig. 6(c) for the undirected networks. In Fig. 11(b), the exponents $\alpha_{G}$ are those in Fig. 3(b) for the directed networks and Fig. 3(d) for the undirected networks, while exponents $\alpha_{P}$ are those in Fig. 6(b) for the directed networks and Fig. 6(d) for the undirected networks. It shows that the data points fall around the line $\alpha_{G} \mathcal{K}=\alpha_{P}$, clsoe to the diagonal.

If we perform linear regression of the data points for each of the cases in Fig. 11, the four slopes are $0.897,0.892,0.889$ and 0.923 , respectively. They are all less than 1 as predicted by Eq. (14). This deviation is partly due to the fact that the derivation of Eq. (14) does not consider the impact of $d_{i j}^{2 \alpha_{1}}$. 

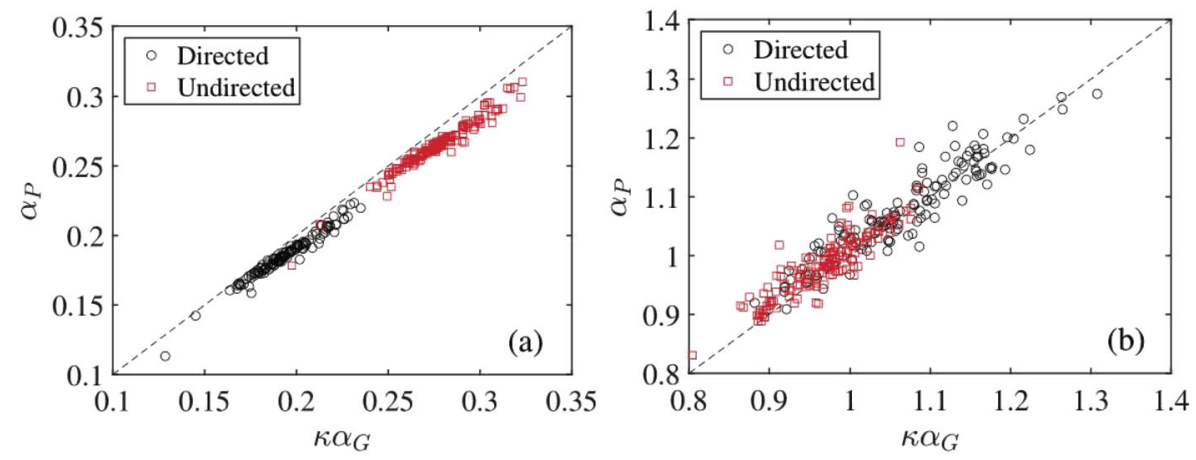

Figure 11 Testing the relationship $\alpha_{G} \kappa=\alpha_{p}$. The data for the dates during 1 February 2019 to 9 February 2019 are excluded. (a) Exponents $\alpha_{G}$ are those in Fig. 3(a) for the directed networks and Fig. 3(c) for the undirected networks, while exponents $\alpha_{P}$ are those in Fig. 6(a) for the directed networks and Fig. 6(c) for the undirected networks. (b) Exponents $\alpha_{G}$ are those in Fig. 3(b) for the directed networks and Fig. 3(d) for the undirected networks, while exponents $\alpha_{p}$ are those in Fig. 6(b) for the directed networks and Fig. 6(d) for the undirected networks
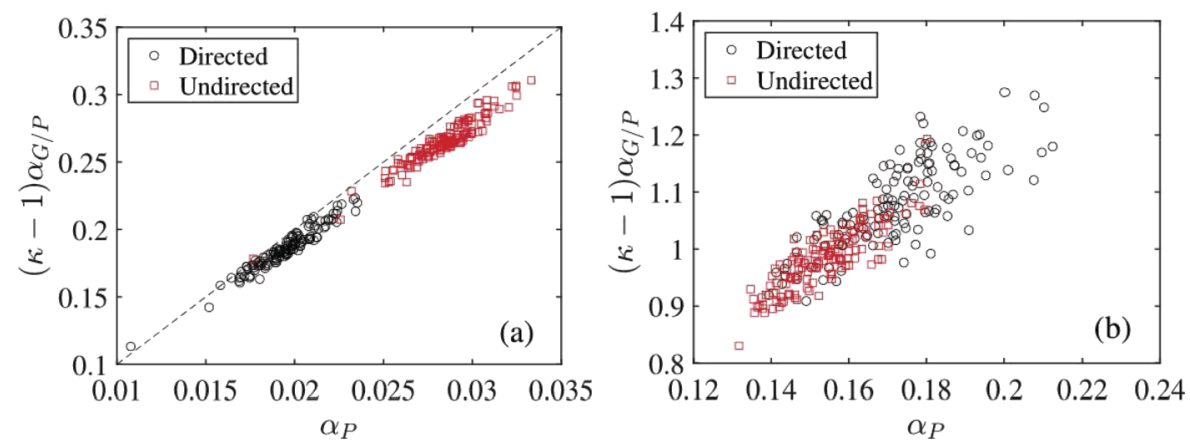

Figure 12 Testing the relationship $(\kappa-1) \alpha_{G / P}=\alpha_{P}$. The data for the dates during 1 February 2019 to 9 February 2019 are excluded. (a) Exponents $\alpha_{G / P}$ are those in Fig. 9(a) for the directed networks and Fig. 9(c) for the undirected networks, while exponents $\alpha_{p}$ are those in Fig. 6(a) for the directed networks and Fig. 6(c) for the undirected networks. (b) Exponents $\alpha_{G / P}$ are those in Fig. 9(b) for the directed networks and Fig. 9(d) for the undirected networks, while exponents $\alpha_{p}$ are those in Fig. $6(\mathrm{~b})$ for the directed networks and Fig. $6(\mathrm{~d})$ for the undirected networks

\subsubsection{Exponents of per capita GDP and population}

From Eq. (10), we have $F_{i j} \sim\left[\left(G_{i} / P_{i}\right)\left(G_{j} / P_{j}\right)\right]_{G / P}^{\alpha}$. From Eq. (8), we have $F_{i j} \sim\left(P_{i} P_{j}\right)_{P}^{\alpha}$. From Eq. (11), we have $W_{i j} \sim\left[\left(G_{i} / P_{i}\right)\left(G_{j} / P_{j}\right)\right]_{G / P}^{\alpha}$. From Eq. (9), we have $W_{i j} \sim\left(P_{i} P_{j}\right)_{P}^{\alpha}$. Combining Eq. (12) and these two gravity laws for GDP and population, it is easy to obtain that

$$
(\kappa-1) \alpha_{G / P}=\alpha_{P},
$$

where $\alpha_{G}$ is $\alpha_{1}$ in the gravity model for GDP and $\alpha_{P}$ is $\alpha_{1}$ in the gravity model for population.

We test the relationship (15) with the exponents of the daily transportation networks. The data for the dates during 1 February 2019 to 9 February 2019 are excluded because their corresponding transportation networks do not exhibit the gravity law. In Fig. 12(a), the exponents $\alpha_{G / P}$ are those in Fig. 9(a) for the directed networks and Fig. 9(c) for the undirected networks, while the exponents $\alpha_{P}$ are those in Fig. 6(a) for the directed net- 
works and Fig. 6(c) for the undirected networks. In Fig. 12, the exponents $\alpha_{G / P}$ are those in Fig. 9(b) for the directed networks and Fig. 9(d) for the undirected networks, while the exponents $\alpha_{P}$ are those in Fig. 6(b) for the directed networks and Fig. 6(d) for the undirected networks.

It shows that the data points in each plot fall around a straight line. However, they are far away from $(\kappa-1) \alpha_{G / P}=\alpha_{P}$. This deviation is partly due to the fact that the derivation of Eq. (15) does not consider the impact of $d_{i j}^{2 \alpha_{1}}$. More importantly, it shows that there are other factors influencing the transportation flow. In other words, the underlying "true model" would be more complicated than the gravity law studied in this work.

\section{The modified gravity model}

In the perspective of directed complex networks, the modified gravity model states that the edge flow $F_{i j}$ between two nodes $i$ and $j$ takes the form of

$$
F_{i j}=C M_{i}^{\alpha_{1}} M_{j}^{\alpha_{2}} / d_{i j}^{\alpha_{3}}
$$

for $i \neq j$, where $C$ is a constant, $d_{i j}$ stands for the distance between $i$ and $j$, and $M_{i}$ and $M_{j}$ stand respectively for the economic dimensions of the two nodes $i$ and $j$ that are being measured. For undirected transportation networks, we have

$$
W_{i j}=C M_{i}^{\alpha_{1}} M_{j}^{\alpha_{2}} / d_{i j}^{\alpha_{3}}
$$

for $j>i$. In our analysis, we consider $M$ to be GDP $(G)$, population $(P)$ and per capita GDP $(G / P)$, respectively.

\subsection{GDP in the modified gravity law}

\subsubsection{Whole transportation networks}

When $M$ in Eq. (16) stands for GDP, the regression equation of the gravity law reads

$$
\lg F_{i j}=\alpha_{0}+\alpha_{1} \lg G_{i}+\alpha_{2} \lg G_{j}-\alpha_{3} \lg d_{i j}+\epsilon,
$$

where $\epsilon$ is the error term. We obtain that $\alpha_{0}=1.171 \pm 0.063, \alpha_{1}=0.621 \pm 0.008, \alpha_{2}=$ $0.637 \pm 0.009$, and $\alpha_{3}=1.191 \pm 0.013$, where the adjusted $R^{2}$ statistic is 0.477 , the $F$ statistic and $p$-value for the full model are respectively 28,447 and 0.000 , and an estimate of the error variance is 0.352 . Figure 13 (a) shows the scatter plot of $F_{i j}$ with respect to $G_{i}^{\hat{\alpha}_{1}} G_{j}^{\hat{\alpha}_{2}} d_{i j}^{\hat{\alpha}_{3}}$ for the directed network. We bin the data with respect to $F$ and illustrate $F_{i j}$ against $G_{i}^{\hat{\alpha}_{1}} G_{j}^{\hat{\alpha}_{2}} d_{i j}^{\hat{\alpha}_{3}}$ in Fig. 13(b). We observe that there is a power-law dependence when $F$ is greater than about 30:

$$
F_{i j}=\alpha_{0}+\alpha_{1} \lg \left[G_{i}^{\hat{\alpha}_{1}} G_{j}^{\hat{\alpha}_{2}} / d_{i j}^{\hat{\alpha}_{3}}\right]+\epsilon
$$

A regression shows that $\alpha_{0}=1.153 \pm 0.031$ and $\alpha_{1}=1.578 \pm 0.028$ with their $p$-values being 0.0000 and 0.0000 . The adjusted $R^{2}$ statistic is 0.995 , and the $F$ statistic and $p$-value for the full model are respectively 3258 and 0.0000 .

When $M$ in Eq. (17) stands for GDP, the regression equation of the gravity law reads

$$
\lg W_{i j}=\alpha_{0}+\alpha_{1} \lg G_{i}+\alpha_{2} \lg G_{j}-\alpha_{3} \lg d_{i j}+\epsilon .
$$



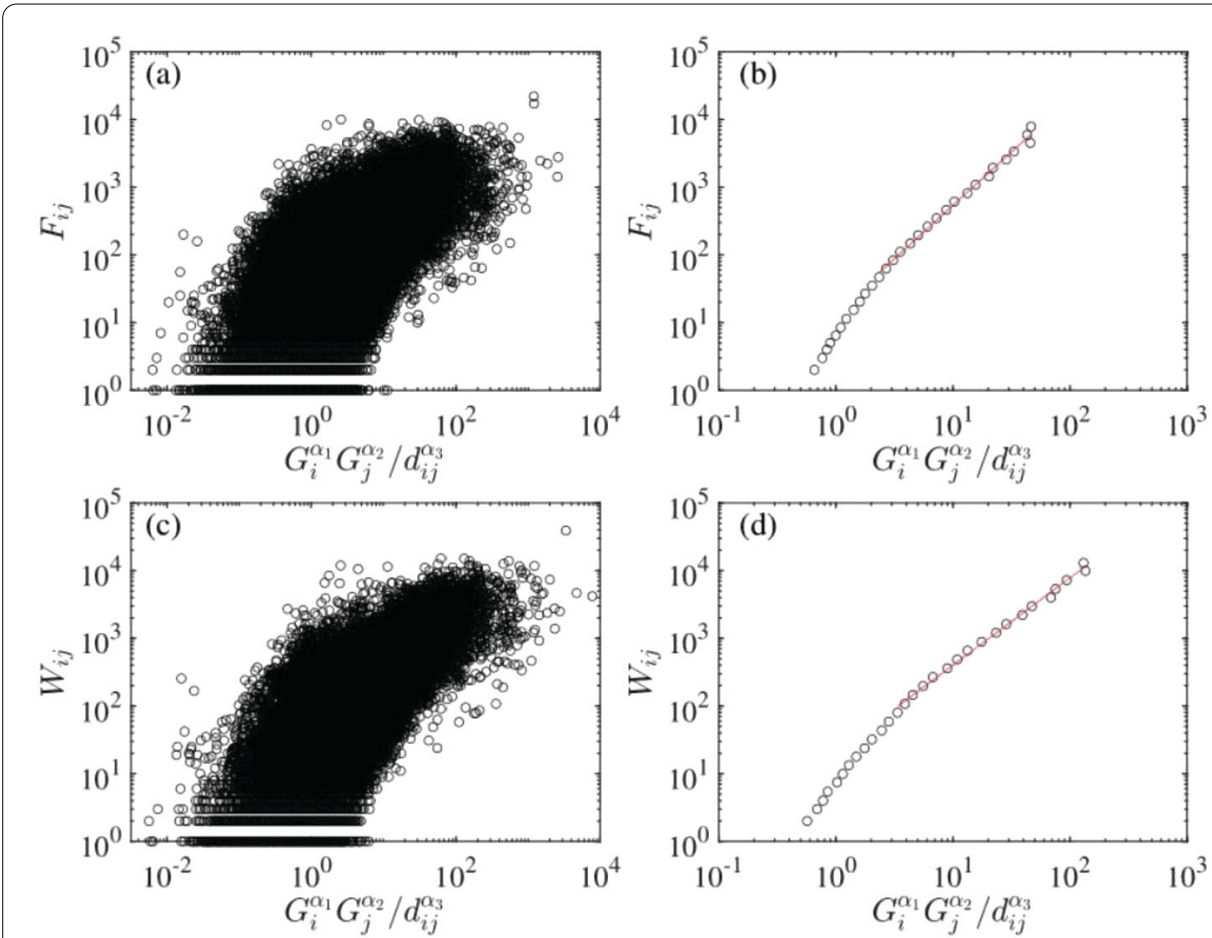

Figure 13 Testing the modified gravity law with GDP for the directed and undirected freight highway transportation networks constructed over five months. (a) Scatter plot of $F_{i j}$ with respect to $G_{i}^{\alpha_{1}} G_{j}^{\alpha_{2}} / d_{i j}^{\alpha_{3}}$ for the directed network. (b) Binning plot of $F_{i j}$ with respect to $G_{i}^{\alpha_{1}} G_{j}^{\alpha_{2}} / d_{i j}^{\alpha_{3}}$ for the directed network. (c) Scatter plot of $W_{i j}$ with respect to $G_{i}^{\alpha_{1}} G_{j}^{\alpha_{2}} / d_{i j}^{\alpha_{3}}$ for the undirected network. (d) Binning plot of $W_{i j}$ with respect to $G_{i}^{\alpha_{1}} G_{j}^{\alpha_{2}} / d_{i j}^{\alpha_{3}}$ for the undirected network

We obtain that $\alpha_{0}=1.273 \pm 0.081, \alpha_{1}=0.743 \pm 0.011, \alpha_{2}=0.649 \pm 0.011$, and $\alpha_{3}=$ $1.255 \pm 0.016$, where the adjusted $R^{2}$ statistic is 0.568 , the $F$ statistic and $p$-value for the full model are respectively 22,379 and 0.000, and an estimate of the error variance is 0.308 . Figure 13(c) shows the scatter plot of $W_{i j}$ with respect to $G_{i}^{\hat{\alpha}_{1}} G_{j}^{\hat{\alpha}_{2}} d_{i j}^{\hat{\alpha}_{3}}$ for the undirected network. We bin the data with respect to $F$ and illustrate $F_{i j}$ against $G_{i}^{\hat{\alpha}_{1}} G_{j}^{\hat{\alpha}_{2}} d_{i j}^{\hat{\alpha}_{3}}$ in Fig. 13(d). We observe that there is a power-law dependence when $F$ is greater than about 30:

$$
W_{i j}=\alpha_{0}+\alpha_{1} \lg \left[G_{i}^{\hat{\alpha}_{1}} G_{j}^{\hat{\alpha}_{2}} / d_{i j}^{\hat{\alpha}_{3}}\right]+\epsilon
$$

We bin the data with respect to $W$ and illustrate the results in Fig. 13(d). We observe that there is a power-law dependence when $W$ is greater than about 200. A regression shows that $\alpha_{0}=1.314 \pm 0.030$ and $\alpha_{1}=1.289 \pm 0.021$ with the $p$-values being 0.0000 and 0.0000 . The adjusted $R^{2}$ statistic is 0.996 , and the $F$ statistic and $p$-value for the full model are respectively 3791 and 0.0000 .

We find that, for the scatter plots, the adjusted $R^{2}$ statistic for the undirected HFTN $(0.568)$ is greater than that for the directed HFTN (0.477). This result is visible in Fig. 13, which shows that the scatter plot is thinner for $W_{i j}$ when compared with the one for $F_{i j}$.

\subsubsection{Daily transportation networks}

We now test the modified gravity law with daily directed and undirected freight highway transportation networks. We find that most of the daily networks exhibit the modified 

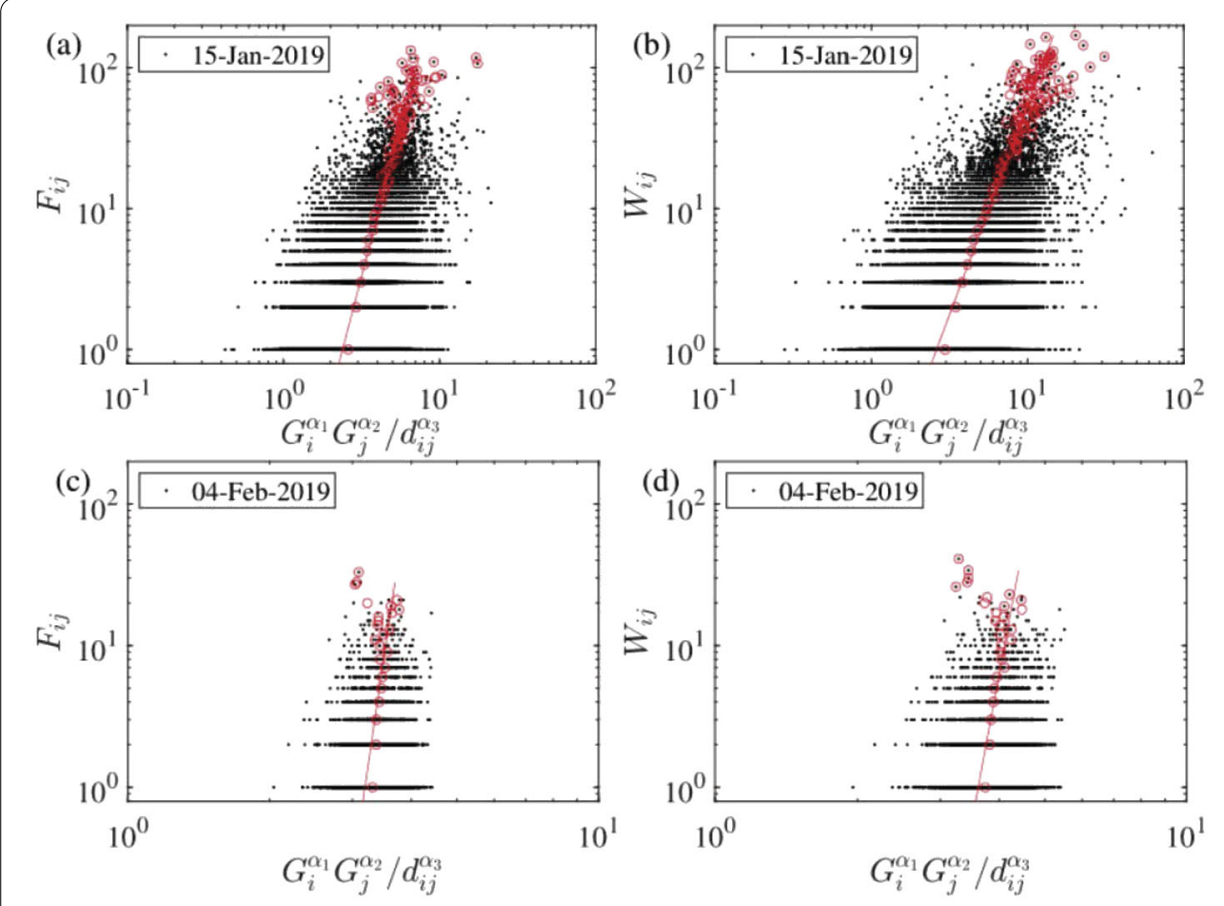

Figure 14 Testing the modified gravity law with GDP for the daily directed and undirected freight highway transportation networks. (a) The directed network on 15 January 2019. (b) The directed network on 15 January 2019. (c) The directed network on 4 February 2019. (d) The undirected network on 4 February 2019

gravity law. As an example, the results for the directed network on 15 January 2019 are illustrated in Fig. 14(a). Regression of Eq. (18) for the scatter data points gives that $\alpha_{0}=$ $-0.119 \pm 0.062, \alpha_{1}=0.242 \pm 0.010, \alpha_{2}=0.202 \pm 0.010$, and $\alpha_{3}=0.347 \pm 0.012$, where the adjusted $R^{2}$ statistic is 0.195 , the $F$ statistic and $p$-value for the full model are respectively 2315 and 0.000, and an estimate of the error variance is 0.128. Regression of Eq. (19) for the binning data points shows that $\alpha_{0}=-1.604 \pm 0.233$ and $\alpha_{1}=4.240 \pm 0.370$, where the adjusted $R^{2}$ statistic is 0.957 , the $F$ statistic and $p$-value for the full model are respectively 556 and 0.000 , and an estimate of the error variance is 0.006 . The results for the undirected network on 15 January 2019 are illustrated in Fig. 14(b). Regression of Eq. (20) for the scatter data points gives that $\alpha_{0}=-0.139 \pm 0.078, \alpha_{1}=0.333 \pm 0.013, \alpha_{2}=0.274 \pm 0.012$, and $\alpha_{3}=0.482 \pm 0.016$, where the adjusted $R^{2}$ statistic is 0.286 , the $F$ statistic and $p$-value for the full model are respectively 2815 and 0.000 , and an estimate of the error variance is 0.141. Regression of Eq. (21) for the binning data points shows that $\alpha_{0}=-1.241 \pm 0.180$ and $\alpha_{1}=2.977 \pm 0.233$, where the adjusted $R^{2}$ statistic is 0.965 , the $F$ statistic and $p$-value for the full model are respectively 694 and 0.000 , and an estimate of the error variance is 0.005 .

However, we find that the daily networks around the Chinese New Year (5 February 2019) do not exhibit the gravity law. The transportation flow decreased significantly during the Spring Festival because most of the truck drivers returned home to gather with their families and most companies were also closed. As an example, the results for the directed network on 4 February 2019 are illustrated in Fig. 14(c). Regression of Eq. (18) for the scatter data points gives that $\alpha_{0}=-0.342 \pm 0.106, \alpha_{1}=0.067 \pm 0.017, \alpha_{2}=0.049 \pm 0.017$, 
and $\alpha_{3}=-0.041 \pm 0.021$, where the adjusted $R^{2}$ statistic is 0.022 , the $F$ statistic and $p$-value for the full model are respectively 36.6 and 0.000 , and an estimate of the error variance is 0.074. Regression of Eq. (19) for the binning data points shows that $\alpha_{0}=-11.425 \pm 10.233$ and $\alpha_{1}=22.681 \pm 19.102$, where the adjusted $R^{2}$ statistic is 0.412 , the $F$ statistic and $p$ value for the full model are respectively 6.999 and 0.024 , and an estimate of the error variance is 0.070 . The results for the undirected network on 15 January 2019 are illustrated in Fig. 14(d). Regression of Eq. (20) for the scatter data points gives that $\alpha_{0}=-0.369 \pm$ $0.118, \alpha_{1}=0.085 \pm 0.019, \alpha_{2}=0.077 \pm 0.019$, and $\alpha_{3}=-0.006 \pm 0.023$, where the adjusted $R^{2}$ statistic is 0.032 , the $F$ statistic and $p$-value for the full model are respectively 48.8 and 0.000 , and an estimate of the error variance is 0.084 . Regression of Eq. (21) for the binning data points shows that $\alpha_{0}=-9.981 \pm 3.990$ and $\alpha_{1}=17.909 \pm 6.637$, where the adjusted $R^{2}$ statistic is 0.742 , the $F$ statistic and $p$-value for the full model are respectively 34.563 and 0.000 , and an estimate of the error variance is 0.032 . The two adjusted $R^{2}$ statistics for the scatter data are close to zero, implying that the term $G_{i}^{\alpha_{1}} G_{j}^{\alpha_{2}} / d_{i j}^{\alpha_{3}}$ does not have explanatory power for the transportation flow $F_{i j}$ or $W_{i j}$ and the modified gravity law is absent.

In Fig. 15, we present the evolution of the exponents $\alpha_{1}$ of daily directed and undirected freight highway transportation networks. In each case, the exponent fluctuates roughly around a constant. The cone peak or valley corresponds to the dates around the Spring Festival during which the gravity law does not hold.

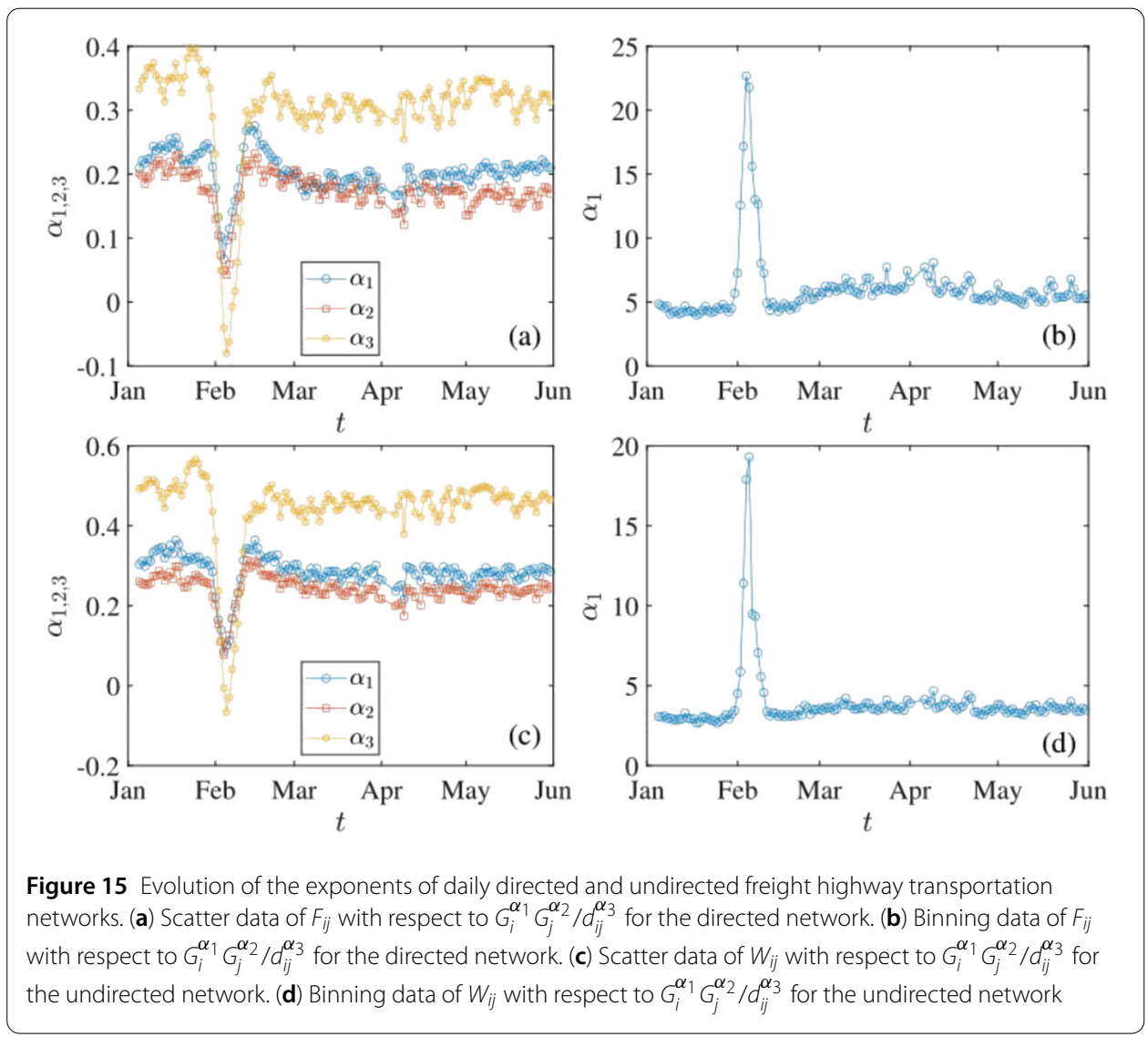




\subsection{Population in the modified gravity law}

\subsubsection{Whole transportation networks}

When $M$ in Eq. (16) stands for population, the regression equation of the gravity law reads

$$
\lg F_{i j}=\alpha_{0}+\alpha_{1} \lg P_{i}+\alpha_{2} \lg P_{j}-\alpha_{3} \lg d_{i j}+\epsilon,
$$

where $\epsilon$ is the error term. We obtain that $\alpha_{0}=1.409 \pm 0.066, \alpha_{1}=0.789 \pm 0.011, \alpha_{2}=$ $0.721 \pm 0.012$, and $\alpha_{3}=1.192 \pm 0.013$, where the adjusted $R^{2}$ statistic is 0.446 , the $F$ statistic and $p$-value for the full model are respectively 25,128 and 0.000, and an estimate of the error variance is 0.372 . Figure 16 (a) shows the scatter plot of $F_{i j}$ with respect to $P_{i}^{\hat{\alpha}_{1}} P_{j}^{\hat{\alpha}_{2}} d_{i j}^{\hat{\alpha}_{3}}$ for the directed network. We bin the data with respect to $F$ and illustrate $F_{i j}$ against $P_{i}^{\hat{\alpha}_{1}} P_{j}^{\hat{\alpha}_{2}} d_{i j}^{\hat{\alpha}_{3}}$ in Fig. 16(b). We observe that there is a power-law dependence when $F$ is greater than about 30:

$$
F_{i j}=\alpha_{0}+\alpha_{1} \lg \left[P_{i}^{\hat{\alpha}_{1}} P_{j}^{\hat{\alpha}_{2}} / d_{i j}^{\hat{\alpha}_{3}}\right]+\epsilon
$$

A regression shows that $\alpha_{0}=1.539 \pm 0.024$ and $\alpha_{1}=1.639 \pm 0.028$ with their $p$-values being 0.0000 and 0.0000 . The adjusted $R^{2}$ statistic is 0.995 , and the $F$ statistic and $p$-value for the full model are respectively 3453.6 and 0.0000 .
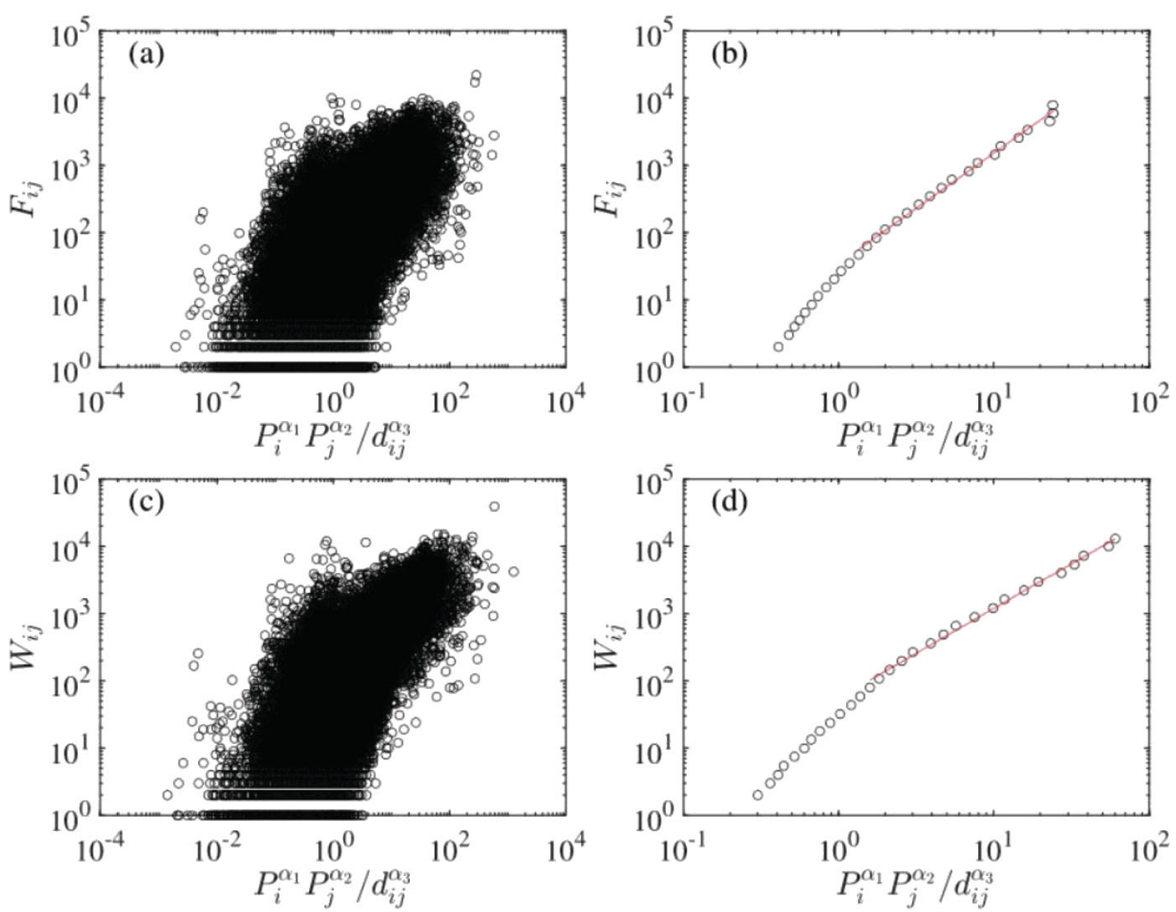

Figure 16 Testing the modified gravity law with population for the directed and undirected freight highway transportation networks constructed over five months. (a) Scatter plot of $F_{i j}$ with respect to $P_{i}^{\alpha_{1}} P_{j}^{\alpha_{2}} / d_{i j}^{\alpha_{3}}$ for the directed network. (b) Binning plot of $F_{i j}$ with respect to $P_{i}^{\alpha_{1}} P_{j}^{\alpha_{2}} / d_{i j}^{\alpha_{3}}$ for the directed network. (c) Scatter plot of $W_{i j}$ with respect to $P_{i}^{\alpha_{1}} P_{j}^{\alpha_{2}} / d_{i j}^{\alpha_{3}}$ for the undirected network. (d) Binning plot of $W_{i j}$ with respect to $P_{i}^{\alpha_{1}} P_{j}^{\alpha_{2}} / d_{i j}^{\alpha_{3}}$ for the undirected network 
When $M$ in Eq. (17) stands for population, the regression equation of the gravity law reads

$$
\lg W_{i j}=\alpha_{0}+\alpha_{1} \lg P_{i}+\alpha_{2} \lg P_{j}-\alpha_{3} \lg d_{i j}+\epsilon .
$$

We obtain that $\alpha_{0}=1.593 \pm 0.085, \alpha_{1}=0.868 \pm 0.014, \alpha_{2}=0.797 \pm 0.015$, and $\alpha_{3}=$ $1.271 \pm 0.017$, where the adjusted $R^{2}$ statistic is 0.531 , the $F$ statistic and $p$-value for the full model are respectively 19,295 and 0.000 , and an estimate of the error variance is 0.334 . Figure 16(c) shows the scatter plot of $W_{i j}$ with respect to $P_{i}^{\hat{\alpha}_{1}} P_{j}^{\hat{\alpha}_{2}} d_{i j}^{\hat{\alpha}_{3}}$ for the undirected network. We bin the data with respect to $F$ and illustrate $F_{i j}$ against $P_{i}^{\hat{\alpha}_{1}} P_{j}^{\hat{\alpha}_{2}} d_{i j}^{\hat{\alpha}_{3}}$ in Fig. $16(\mathrm{~d})$. We observe that there is a power-law dependence when $F$ is greater than about 30:

$$
W_{i j}=\alpha_{0}+\alpha_{1} \lg \left[P_{i}^{\hat{\alpha}_{1}} P_{j}^{\hat{\alpha}_{2}} / d_{i j}^{\hat{\alpha}_{3}}\right]+\epsilon \text {. }
$$

We bin the data with respect to $W$ and illustrate the results in Fig. 16(d). We observe that there is a power-law dependence when $W$ is greater than about 200. A regression shows that $\alpha_{0}=1.741 \pm 0.024$ and $\alpha_{1}=1.333 \pm 0.022$ with the $p$-values being 0.0000 and 0.0000 . The adjusted $R^{2}$ statistic is 0.995 , and the $F$ statistic and $p$-value for the full model are respectively 3631 and 0.0000 .

We find that, for the scatter plots, the adjusted $R^{2}$ statistic for the undirected HFTN (0.531) is greater than that for the directed HFTN (0.446). This result is visible in Fig. 16, which shows that the scatter plot is thinner for $W_{i j}$ when compared with the one for $F_{i j}$.

\subsubsection{Daily transportation networks}

We now test the modified gravity law with daily directed and undirected freight highway transportation networks. We find that most of the daily networks exhibit the modified gravity law. As an example, the results for the directed network on 15 January 2019 are illustrated in Fig. 17(a). Regression of Eq. (22) for the scatter data points gives that $\alpha_{0}=$ $-0.244 \pm 0.066, \alpha_{1}=0.353 \pm 0.014, \alpha_{2}=0.251 \pm 0.014$, and $\alpha_{3}=0.336 \pm 0.013$, where the adjusted $R^{2}$ statistic is 0.192 , the $F$ statistic and $p$-value for the full model are respectively 2278 and 0.000 , and an estimate of the error variance is 0.129. Regression of Eq. (23) for the binning data points shows that $\alpha_{0}=-2.188 \pm 0.319$ and $\alpha_{1}=4.334 \pm 0.426$, where the adjusted $R^{2}$ statistic is 0.946 , the $F$ statistic and $p$-value for the full model are respectively 439 and 0.000 , and an estimate of the error variance is 0.007 . The results for the undirected network on 15 January 2019 are illustrated in Fig. 17(b). Regression of Eq. (24) for the scatter data points gives that $\alpha_{0}=-0.189 \pm 0.083, \alpha_{1}=0.420 \pm 0.017, \alpha_{2}=0.361 \pm 0.017$, and $\alpha_{3}=0.470 \pm 0.016$, where the adjusted $R^{2}$ statistic is 0.271 , the $F$ statistic and $p$-value for the full model are respectively 2616 and 0.000 , and an estimate of the error variance is 0.144. Regression of Eq. (25) for the binning data points shows that $\alpha_{0}=-1.427 \pm 0.210$ and $\alpha_{1}=3.046 \pm 0.257$, where the adjusted $R^{2}$ statistic is 0.960 , the $F$ statistic and $p$-value for the full model are respectively 596 and 0.000 , and an estimate of the error variance is 0.006 .

However, we find that the daily networks around the Chinese New Year (5 February 2019) do not exhibit the gravity law. The transportation flow decreased significantly during the Spring Festival because most of the truck drivers returned home to gather with their families and most companies were also closed. As an example, the results for the directed 

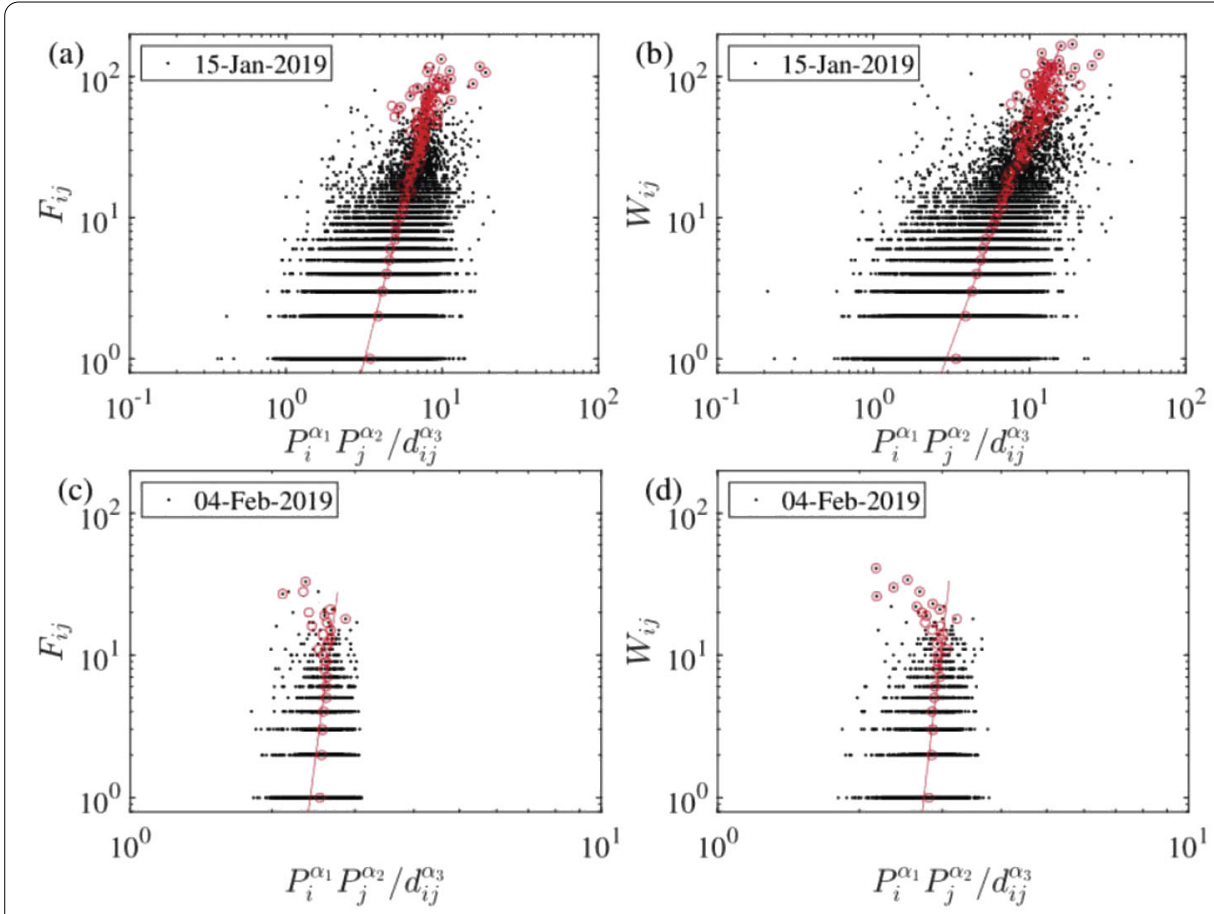

Figure 17 Testing the modified gravity law with population for the daily directed and undirected freight highway transportation networks. (a) The directed network on 15 January 2019. (b) The directed network on 15 January 2019. (c) The directed network on 4 February 2019. (d) The undirected network on 4 February 2019

network on 4 February 2019 are illustrated in Fig. 17(c). Regression of Eq. (22) for the scatter data points gives that $\alpha_{0}=-0.223 \pm 0.114, \alpha_{1}=0.082 \pm 0.025, \alpha_{2}=0.026 \pm 0.024$, and $\alpha_{3}=-0.037 \pm 0.021$, where the adjusted $R^{2}$ statistic is 0.012 , the $F$ statistic and $p$-value for the full model are respectively 20.3 and 0.000 , and an estimate of the error variance is 0.075. Regression of Eq. (23) for the binning data points shows that $\alpha_{0}=-9.526 \pm 13.747$ and $\alpha_{1}=24.918 \pm 33.416$, where the adjusted $R^{2}$ statistic is 0.216 , the $F$ statistic and $p$ value for the full model are respectively 2.76 and 0.128 , and an estimate of the error variance is 0.093 . The results for the undirected network on 15 January 2019 are illustrated in Fig. 17(d). Regression of Eq. (24) for the scatter data points gives that $\alpha_{0}=-0.242 \pm 0.128$, $\alpha_{1}=0.097 \pm 0.027, \alpha_{2}=0.066 \pm 0.027$, and $\alpha_{3}=-0.005 \pm 0.024$, where the adjusted $R^{2}$ statistic is 0.017 , the $F$ statistic and $p$-value for the full model are respectively 24.7 and 0.000 , and an estimate of the error variance is 0.085 . Regression of Eq. (25) for the binning data points shows that $\alpha_{0}=-12.333 \pm 5.064$ and $\alpha_{1}=28.166 \pm 10.873$, where the adjusted $R^{2}$ statistic is 0.726 , the $F$ statistic and $p$-value for the full model are respectively 31.854 and 0.000 , and an estimate of the error variance is 0.034 . The two adjusted $R^{2}$ statistics for the scatter data are close to zero, implying that the term $P_{i}^{\alpha_{1}} P_{j}^{\alpha_{2}} / d_{i j}^{\alpha_{3}}$ does not have explanatory power for the transportation flow $F_{i j}$ or $W_{i j}$ and the modified gravity law is absent.

In Fig. 18, we present the evolution of the exponents $\alpha_{1}$ of daily directed and undirected freight highway transportation networks. In each case, the exponent fluctuates roughly around a constant. The cone peak or valley corresponds to the dates around the Spring Festival during which the gravity law does not hold. 


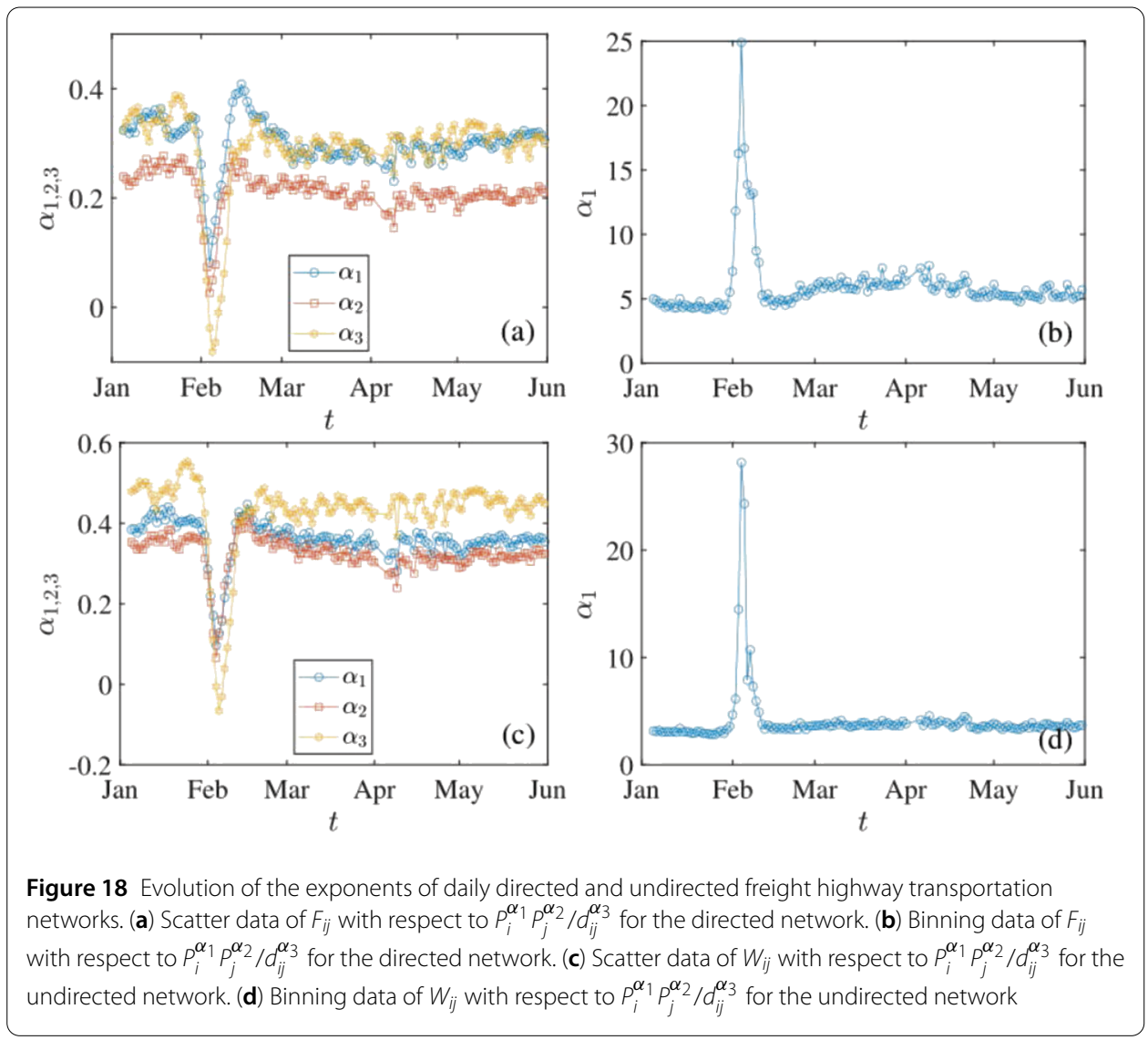

\subsection{Per capita GDP in the modified gravity law}

\subsubsection{Whole transportation networks}

When $M$ in Eq. (16) stands for per capita GDP, the regression equation of the modified gravity law reads

$$
\lg F_{i j}=\alpha_{0}+\alpha_{1} \lg \left(G_{i} / P_{i}\right)+\alpha_{2} \lg \left(G_{j} / P_{j}\right)-\alpha_{3} \lg d_{i j}+\epsilon,
$$

where $\epsilon$ is the error term. We obtain that $\alpha_{0}=4.984 \pm 0.050, \alpha_{1}=0.544 \pm 0.018, \alpha_{2}=$ $0.681 \pm 0.019$, and $\alpha_{3}=1.385 \pm 0.014$, where the adjusted $R^{2}$ statistic is 0.330 , the $F$ statistic and $p$-value for the full model are respectively 15,365 and 0.000, and an estimate of the error variance is 0.451 . Figure 19(a) shows the scatter plot of $F_{i j}$ with respect to $\left(G_{i} / P_{i}\right)^{\hat{\alpha}_{1}}\left(G_{j} / P_{j}\right)^{\hat{\alpha}_{2}} / d_{i j}^{\hat{\alpha}_{3}}$ for the directed network. We bin the data with respect to $F$ and illustrate $F_{i j}$ against $\left(G_{i} / P_{i}\right)^{\hat{\alpha}_{1}}\left(G_{j} / P_{j}\right)^{\hat{\alpha}_{2}} / d_{i j}^{\hat{\alpha}_{3}}$ in Fig. 19(b). We observe that there is a power-law dependence when $F$ is greater than about 30:

$$
F_{i j}=\alpha_{0}+\alpha_{1} \lg \left[\left(G_{i} / P_{i}\right)^{\hat{\alpha}_{1}}\left(G_{j} / P_{j}\right)^{\hat{\alpha}_{2}} / d_{i j}^{\hat{\alpha}_{3}}\right]+\epsilon .
$$

A regression shows that $\alpha_{0}=9.317 \pm 0.158$ and $\alpha_{1}=2.191 \pm 0.053$ with their $p$-values being 0.0000 and 0.0000 . The adjusted $R^{2}$ statistic is 0.990 , and the $F$ statistic and $p$-value for the full model are respectively 1736 and 0.0000 . 

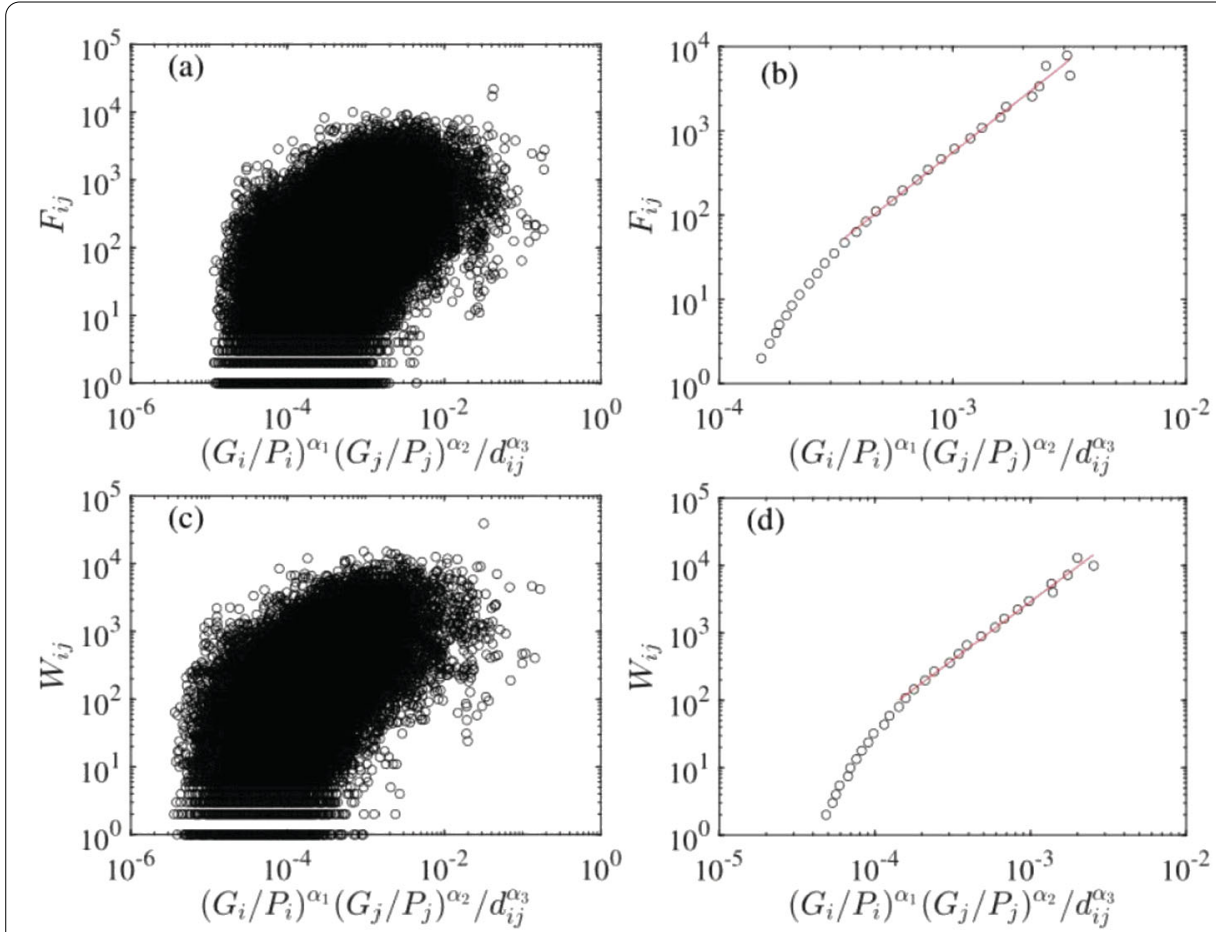

Figure 19 Testing the modified gravity law with population for the directed and undirected freight highway transportation networks constructed over five months. (a) Scatter plot of $F_{i j}$ with respect to

$\left(G_{j} / P_{j}\right)^{\alpha_{1}}\left(G_{j} / P_{j}\right)^{\alpha_{2}} / d_{i j}^{\alpha_{3}}$ for the directed network. (b) Binning plot of $F_{i j}$ with respect to $\left(G_{j} / P_{j}\right)^{\alpha_{1}}\left(G_{j} / P_{j}\right)^{\alpha_{2}} / d_{i j}^{\alpha_{3}}$ for the directed network. (c) Scatter plot of $W_{i j}$ with respect to $\left(G_{j} / P_{i}\right)^{\alpha_{1}}\left(G_{j} / P_{j}\right)^{\alpha_{2}} / d_{i j}^{\alpha_{3}}$ for the undirected network. (d) Binning plot of $W_{i j}$ with respect to $\left(G_{j} / P_{i}\right)^{\alpha_{1}}\left(G_{j} / P_{j}\right)^{\alpha_{2}} / d_{i j}^{\alpha_{3}}$ for the undirected network

When $M$ in Eq. (17) stands for population, the regression equation of the gravity law reads

$$
\lg W_{i j}=\alpha_{0}+\alpha_{1} \lg \left(G_{i} / P_{i}\right)+\alpha_{2} \lg \left(G_{j} / P_{j}\right)-\alpha_{3} \lg d_{i j}+\epsilon .
$$

We obtain that $\alpha_{0}=5.621 \pm 0.067, \alpha_{1}=0.660 \pm 0.026, \alpha_{2}=0.701 \pm 0.023$, and $\alpha_{3}=$ $1.520 \pm 0.019$, where the adjusted $R^{2}$ statistic is 0.388 , the $F$ statistic and $p$-value for the full model are respectively 10,769 and 0.000 , and an estimate of the error variance is 0.436 . Figure 19 (c) shows the scatter plot of $W_{i j}$ with respect to $\left(G_{i} / P_{i}\right)^{\alpha_{1}}\left(G_{j} / P_{j}\right)^{\alpha_{2}} / d_{i j}^{\alpha_{3}}$ for the undirected network. We bin the data with respect to $F$ and illustrate $F_{i j}$ against $\left(G_{i} / P_{i}\right)^{\hat{\alpha}_{1}}\left(G_{j} / P_{j}\right)^{\hat{\alpha}_{2}} / d_{i j}^{\hat{\alpha}_{3}}$ in Fig. 19(d). We observe that there is a power-law dependence when $F$ is greater than about 30 :

$$
W_{i j}=\alpha_{0}+\alpha_{1} \lg \left[\left(G_{i} / P_{i}\right)^{\hat{\alpha}_{1}}\left(G_{j} / P_{j}\right)^{\hat{\alpha}_{2}} / d_{i j}^{\hat{\alpha}_{3}}\right]+\epsilon .
$$

We bin the data with respect to $W$ and illustrate the results in Fig. 19(d). We observe that there is a power-law dependence when $W$ is greater than about 200. A regression shows that $\alpha_{0}=8.641 \pm 0.140$ and $\alpha_{1}=1.726 \pm 0.043$ with the $p$-values being 0.0000 and 0.0000 . The adjusted $R^{2}$ statistic is 0.990 , and the $F$ statistic and $p$-value for the full model are respectively 1642 and 0.0000 . 
We find that, for the scatter plots, the adjusted $R^{2}$ statistic for the undirected HFTN $(0.388)$ is greater than that for the directed HFTN (0.330). This result is visible in Fig. 19, which shows that the scatter plot is thinner for $W_{i j}$ when compared with the one for $F_{i j}$.

\subsubsection{Daily transportation networks}

We now test the modified gravity law with daily directed and undirected freight highway transportation networks. We find that most of the daily networks exhibit the modified gravity law. As an example, the results for the directed network on 15 January 2019 are illustrated in Fig. 20(a). Regression of Eq. (26) for the scatter data points gives that $\alpha_{0}=$ $1.147 \pm 0.043, \alpha_{1}=0.199 \pm 0.019, \alpha_{2}=0.197 \pm 0.019$, and $\alpha_{3}=0.364 \pm 0.013$, where the adjusted $R^{2}$ statistic is 0.117 , the $F$ statistic and $p$-value for the full model are respectively 1264 and 0.000, and an estimate of the error variance is 0.141. Regression of Eq. (27) for the binning data points shows that $\alpha_{0}=5.689 \pm 0.583$ and $\alpha_{1}=6.644 \pm 0.830$, where the adjusted $R^{2}$ statistic is 0.916 , the $F$ statistic and $p$-value for the full model are respectively 272 and 0.000 , and an estimate of the error variance is 0.012 . The results for the undirected network on 15 January 2019 are illustrated in Fig. 20(b). Regression of Eq. (28) for the scatter data points gives that $\alpha_{0}=1.582 \pm 0.056, \alpha_{1}=0.271 \pm 0.024, \alpha_{2}=0.272 \pm 0.023$, and $\alpha_{3}=0.509 \pm 0.017$, where the adjusted $R^{2}$ statistic is 0.175 , the $F$ statistic and $p$-value for the full model are respectively 1489 and 0.000 , and an estimate of the error variance is 0.163. Regression of Eq. (29) for the binning data points shows that $\alpha_{0}=5.773 \pm 0.584$ and $\alpha_{1}=4.622 \pm 0.569$, where the adjusted $R^{2}$ statistic is 0.918 , the $F$ statistic and $p$-value for the full model are respectively 280 and 0.000 , and an estimate of the error variance is 0.011 .
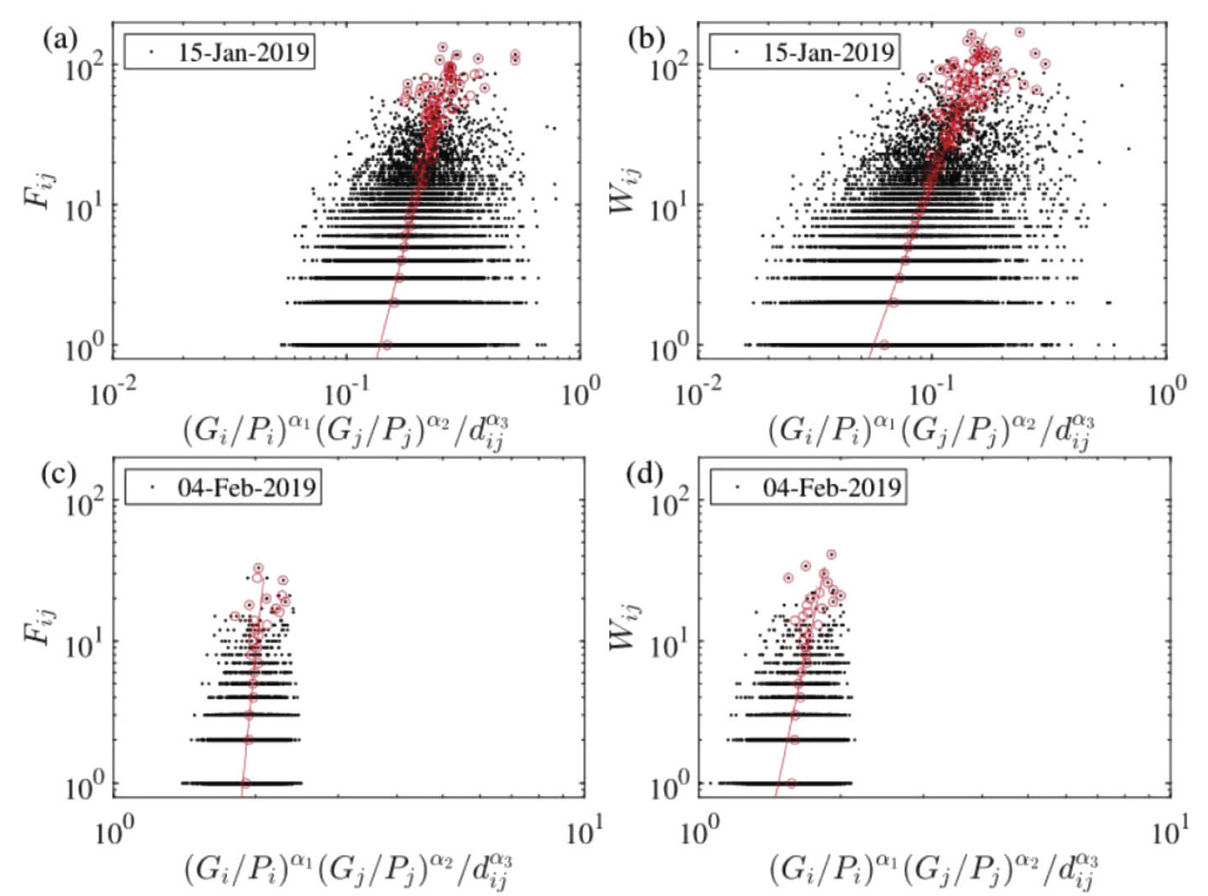

Figure 20 Testing the modified gravity law with per capita GDP for the daily directed and undirected freight highway transportation networks. (a) The directed network on 15 January 2019. (b) The directed network on 15 January 2019. (c) The directed network on 4 February 2019. (d) The undirected network on 4 February 2019 
However, we find that the daily networks around the Chinese New Year (5 February 2019) do not exhibit the gravity law. The transportation flow decreased significantly during the Spring Festival because most of the truck drivers returned home to gather with their families and most companies were also closed. As an example, the results for the directed network on 4 February 2019 are illustrated in Fig. 20(c). Regression of Eq. (26) for the scatter data points gives that $\alpha_{0}=-0.103 \pm 0.071, \alpha_{1}=0.097 \pm 0.032, \alpha_{2}=0.124 \pm 0.032$, and $\alpha_{3}=-0.039 \pm 0.021$, where the adjusted $R^{2}$ statistic is 0.023 , the $F$ statistic and $p$-value for the full model are respectively 37.4 and 0.000 , and an estimate of the error variance is 0.074. Regression of Eq. (27) for the binning data points shows that $\alpha_{0}=-8.942 \pm 4.624$ and $\alpha_{1}=32.579 \pm 15.580$, where the adjusted $R^{2}$ statistic is 0.685 , the $F$ statistic and $p$ value for the full model are respectively 21.7 and 0.001 , and an estimate of the error variance is 0.037 . The results for the undirected network on 15 January 2019 are illustrated in Fig. 20(d). Regression of Eq. (28) for the scatter data points gives that $\alpha_{0}=0.007 \pm 0.079$, $\alpha_{1}=0.122 \pm 0.037, \alpha_{2}=0.156 \pm 0.034$, and $\alpha_{3}=0.004 \pm 0.023$, where the adjusted $R^{2}$ statistic is 0.028 , the $F$ statistic and $p$-value for the full model are respectively 42.6 and 0.000 , and an estimate of the error variance is 0.084 . Regression of Eq. (29) for the binning data points shows that $\alpha_{0}=-2.627 \pm 2.288$ and $\alpha_{1}=15.534 \pm 10.407$, where the adjusted $R^{2}$ statistic is 0.468 , the $F$ statistic and $p$-value for the full model are respectively 10.6 and 0.007 , and an estimate of the error variance is 0.065 . The two adjusted $R^{2}$ statistics for the scatter data are close to zero, implying that the term $\left(G_{i} / P_{i}\right)^{\alpha_{1}}\left(G_{j} / P_{j}\right)^{\alpha_{2}} / d_{i j}^{\alpha_{3}}$ does not have explanatory power for the transportation flow $F_{i j}$ or $W_{i j}$ and the modified gravity law is absent.

In Fig. 21, we present the evolution of the exponents $\alpha_{1}$ of daily directed and undirected freight highway transportation networks. In each case, the exponent fluctuates roughly around a constant. The cone peak or valley corresponds to the dates around the Spring Festival during which the gravity law does not hold.

\section{Summary and discussions}

In this work, we constructed the directed and undirected highway freight transportation networks between 338 Chinese cities using about 15.06 million truck transportation records in five months (2019/01/05-2019/05/31) and tested the traditional and modified gravity laws using GDP, population and per capita GDP as the node strength. It is found that the gravity law holds over about two orders of magnitude for the whole sample, as well as the daily samples, except for the days around the Spring Festival during which the daily sample sizes are significantly small. Accordingly, the daily exponents of the gravity law are stable except during the Spring Festival period. Other than the Spring Festival holiday, there are a number of holidays in China. However, we observe that there show no abnormal phenomena in the time series of $\alpha_{1}$ other than the Spring Festival. These seemingly counterintuitive results are explained by the fact that the Spring Festival is special other than other holidays. It is the tradition that almost all Chinese stayed in other places will return to their hometowns to have dinner with all family members especially parents on the Eve of the Spring Festival. Accordingly, most companies and factories are closed during the Spring Festival for one or two weeks, resulting in a sharp decrease in the demand of freight transportation.

We confirmed that there is a power-law scaling between GDP and population at the city level. We carried out a scaling analysis and obtained the relationship between the expo- 


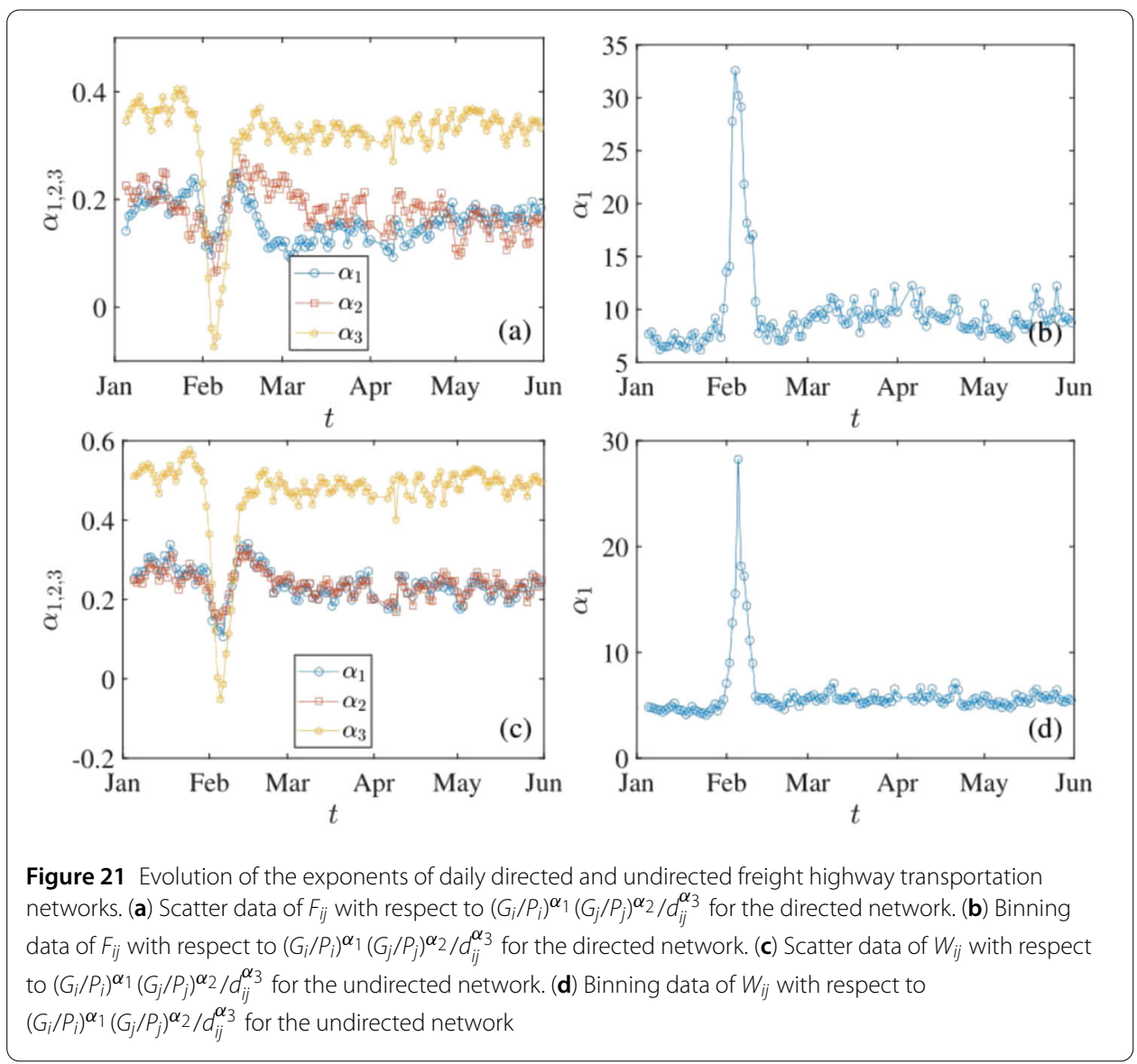

nents of different gravity laws. Using daily transportation networks, the scaling relationship between the GDP-based gravity law and the population-based gravity law has been well verified with mild deviations. The feasible regions for the gravity laws differ from each other (see the figures). However, the scaling relationship could not be confirmed when the per capita GDP-based gravity law is considered. To some extent, this observation is consistent with the fact that the per capita GDP-based gravity law gives significantly smaller adjusted $R^{2}$ values.

The adjusted $R^{2}$ values show that the gravity law has higher explanatory power for the undirected highway freight transportation networks than for the directed highway freight transportation networks. However, the traditional and modified gravity laws have comparable explanatory power according to the fact that their corresponding adjusted $R^{2}$ values are very close to each other. In addition, although the GDP-based and the population based models already have quite high explanatory power for the transportation flows (more than $40 \%$ for directed networks and more than $50 \%$ for undirected networks), additional factors that account for the unexplained components call for further research. One possible direction is to consider the comparative advantage of different cities embedded in the industrial structure of China as well as different demands from different cities.

We note that the fitted parameters or exponents are not general. The literature has shown that the exponents could be different due to different systems (air lines, trains, buses, et al.), different time periods, and different places. In addition, there are other 
newly-proposed important models $[4,6]$ that should be investigated using this huge data base. These interesting problems will be researched in future works.

\section{Funding}

This work was partly supported by the Fundamental Research Funds for the Central Universities.

\section{Abbreviations}

HFTN, stands for highway freight transportation network; GDP, is gross domestic product.

Availability of data and materials

We signed a confidentiality agreement with the transportation company who provided us the data used in this work. Hence the data will not be shared.

Ethics approval and consent to participate

Not applicable.

Competing interests

The authors declare that they have no competing interests.

Consent for publication

Not applicable.

\section{Authors' contributions}

Z-QJ, WY and W-XZ conceived the research. Z-QJ, WY and W-XZ designed the analyses. J-CM compiled the data. LW and W-XZ conducted the analyses. All authors discussed the results. W-XZ wrote the paper. All authors read and approved the final manuscript.

\section{Author details}

${ }^{1}$ School of Business, East China University of Science and Technology, Shanghai, China. ${ }^{2}$ Zhicang Technologies, Beijing, China. ${ }^{3}$ Research Center for Econophysics, East China University of Science and Technology, Shanghai, China.

${ }^{4}$ Department of Mathematics, East China University of Science and Technology, Shanghai, China.

\section{Publisher's Note}

Springer Nature remains neutral with regard to jurisdictional claims in published maps and institutional affiliations.

Received: 13 September 2019 Accepted: 5 December 2019 Published online: 12 December 2019

\section{References}

1. Ravenstein EG (1889) The laws of migration. J R Stat Soc 52(2):241-305. https://doi.org/10.2307/2979333

2. Karemera D, Oguledo VI, Davis B (2000) A gravity model analysis of international migration to North America. Appl Econ 32(13):1745-1755. https://doi.org/10.1080/000368400421093

3. Beine M, Bertoli S, Fernandez-Huertas Moraga J (2016) A practitioners' guide to gravity models of international migration. World Econ 39(4):496-512. https://doi.org/10.1111/twec.12265

4. Simini F, González MC, Maritan A, Barabási A-L (2012) A universal model for mobility and migration patterns. Nature 484(7392):96-100. https://doi.org/10.1038/nature10856

5. Fagiolo G, Santoni G (2016) Revisiting the role of migrant social networks as determinants of international migration flows. Appl Econ Lett 23(3):188-193. https://doi.org/10.1080/13504851.2015.1064072

6. Yan X-Y, Wang W-X, Gao Z-Y, Lai Y-C (2017) Universal model of individual and population mobility on diverse spatial scales. Nat Commun 8:1639. https://doi.org/10.1038/s41467-017-01892-8

7. Hong I, Jung W-S, Jo H-H (2019) Gravity model explained by the radiation model on a population landscape. PLoS ONE 14(6):0218028. https://doi.org/10.1371/journal.pone.0218028

8. Isard W (1954) Location theory and trade theory: short-run analysis. Q J Econ 68(2):305-320. https://doi.org/10.2307/1884452

9. Tinbergen J (1962) Shaping the world economy: suggestions for an international economic policy. Twentieth Century Fund, New York

10. Anderson JE (1979) A theoretical foundation for the gravity equation. Am Econ Rev 69(1):106-116

11. Senior ML (1979) From gravity modelling to entropy maximizing: a pedagogic guide. Prog Hum Geogr 3(2):175-210. https://doi.org/10.1177/030913257900300218

12. Bergstrand JH (1989) The generalized gravity equation, monopolistic competition, and the factor-proportions theory in international trade. Rev Econ Stat 71(1):143-153. https://doi.org/10.2307/1928061

13. Anderson JE, van Wincoop E (2003) Gravity with gravitas: a solution to the border puzzle. Am Econ Rev 93(1):170-192. https://doi.org/10.1257/000282803321455214

14. Fagiolo G (2010) The international-trade network: gravity equations and topological properties. J Econ Interact Coord 5(1):1-25. https://doi.org/10.1007/s11403-010-0061-y

15. Anderson JE (2011) The gravity model. Annu Rev Econ 3:133-160. https://doi.org/10.1146/annurev-economics-111809-125114

16. Duenas M, Fagiolo G (2013) Modeling the international-trade network: a gravity approach. J Econ Interact Coord 8(1):155-178. https://doi.org/10.1007/s11403-013-0108-y 
17. Almog A, Bird R, Garlaschelli D (2019) Enhanced gravity model of trade: reconciling macroeconomic and network models. Front Phys 7:55. https://doi.org/10.3389/fphy.2019.00055

18. Lambiotte R, Blondel VD, de Kerchove C, Huens E, Prieur C, Smoreda Z, Van Dooren P (2008) Geographical dispersal of mobile communication networks. Physica A 387:5317-5325. https://doi.org/10.1016/j.physa.2008.05.014

19. Krings G, Calabrese F, Ratti C, Blondel VD (2009) Urban gravity: a model for inter-city telecommunication flows. J Stat Mech Theory Exp 2009(7):07003. https://doi.org/10.1088/1742-5468/2009/07/L07003

20. Onnela J-P, Arbesman S, González MC, Barabási A-L, Christakis NA (2011) Geographic constraints on social network groups. PLoS ONE 6(4):16939. https://doi.org/10.1371/journal.pone.0016939

21. Palchykov V, Mitrovic M, Jo H-H, Saramäki J, Pan RK (2014) Inferring human mobility using communication patterns. Sci Rep 4:6174. https://doi.org/10.1038/srep06174

22. Blondel VD, Decuyper A, Krings G (2015) A survey of results on mobile phone datasets analysis. EPJ Data Sci 4(1):10 https://doi.org/10.1140/epjds/s13688-015-0046-0

23. Beiro MG, Bravo L, Caro D, Cattuto C, Ferres L, Graells-Garrido E (2018) Shopping mall attraction and social mixing at a city scale. EPJ Data Sci 7(1):28. https://doi.org/10.1140/epjds/s13688-018-0157-5

24. Jung W-S, Wang F-Z, Stanley HE (2008) Gravity model in the Korean highway. Europhys Lett 81(4):48005. https://doi.org/10.1209/0295-5075/81/48005

25. Kwon O, Jung W-S (2012) Intercity express bus flow in Korea and its network analysis. Physica A 391(17):4261-4265. https://doi.org/10.1016/j.physa.2012.03.031

26. Hong I, Jung W-S (2016) Application of gravity model on the Korean urban bus network. Physica A 462:48-55. https://doi.org/10.1016/j.physa.2016.06.055

27. Serrano MA, Boguñá M (2003) Topology of the world trade web. Phys Rev E 68:015101 https://doi.org/10.1103/PhysRevE.68.015101

28. Garlaschelli D, Loffredo MI (2004) Fitness-dependent topological properties of the world trade web. Phys Rev Lett 93:188701. https://doi.org/10.1103/PhysRevLett.93.188701

29. Bettencourt LMA, Lobo J, Helbing D, Kuhnert C, West GB (2007) Growth, innovation, scaling, and the pace of life in cities. Proc Natl Acad Sci USA 104(17):7301-7306. https://doi.org/10.1073/pnas.0610172104

\section{Submit your manuscript to a SpringerOpen ${ }^{0}$ journal and benefit from:}

- Convenient online submission

- Rigorous peer review

- Open access: articles freely available online

- High visibility within the field

- Retaining the copyright to your article

Submit your next manuscript at $\gg$ springeropen.com 